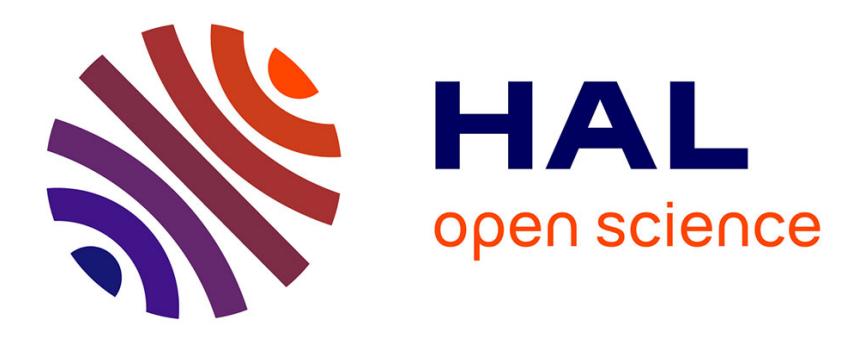

\title{
A discrete formulation of Kirchhoff rods in large-motion dynamics \\ Ivan Giorgio
}

\section{To cite this version:}

Ivan Giorgio. A discrete formulation of Kirchhoff rods in large-motion dynamics. 2020. hal-02436054

\section{HAL Id: hal-02436054 \\ https://hal.science/hal-02436054}

Preprint submitted on 12 Jan 2020

HAL is a multi-disciplinary open access archive for the deposit and dissemination of scientific research documents, whether they are published or not. The documents may come from teaching and research institutions in France or abroad, or from public or private research centers.
L'archive ouverte pluridisciplinaire HAL, est destinée au dépôt et à la diffusion de documents scientifiques de niveau recherche, publiés ou non, émanant des établissements d'enseignement et de recherche français ou étrangers, des laboratoires publics ou privés. 


\title{
A discrete formulation of Kirchhoff rods in large-motion dynamics
}

\author{
Ivan Giorgio'
}

\begin{abstract}
A nonlinear model for the dynamics of a Kirchhoff rod in the three-dimensional space is developed in the framework of a discrete elastic theory. The formulation avoids the use of Euler angles for the orientation of the rod cross-sections to provide a computationally singularity-free parameterization of rotations along the motion trajectories. The material directions related to the principal axes of the crosssections are specified using auxiliary points that must satisfy constraints enforced by the Lagrange multipliers method. A generalization of this approach is presented to take into account Poisson's effect in an orthotropic rod. Numerical simulations are performed to test the presented formulation.
\end{abstract}

\section{Keywords}

Kirchhoff viscoelastic beam; Nonlinear rod; Large displacements and rotations; Discrete formulation; Lagrange multipliers.

\section{Introduction}

This paper is concerned with a discrete formulation of the three-dimensional Kirchhoff beam theory accounting for stretching, bending, and twisting deformations. The approach discussed herein focuses especially on dynamic problems, and aims at overcoming the issues arising when classical representations of orthogonal transformations, such as Euler angles or the angle/axis representation, are being used.

In order to adequately describe the orientation of the cross-sections of a Kirchhoff rod in a spatial motion, a rotation field - in a one-dimensional continuum Eugster et al.

\footnotetext{
${ }^{1}$ International Research Centre on Mathematics and Mechanics of Complex Systems, Italy

Corresponding author:

Ivan Giorgio, Università degli studi dell'Aquila, International Research Centre on Mathematics and Mechanics of Complex Systems, 67100, L'Aquila, Italy.

Email: ivan.giorgio@univaq.it
} 
(2014); Greco and Cuomo (2014a); Luongo and Zulli (2013); Pideri and Seppecher (2006); Steigmann and Faulkner (1993) — or a finite sequence of rotations — in a discrete formulation Jawed et al. (2018); Bergou et al. (2008); Turco (2018) - is required. Regrettably, the set of variables parametrizing the tensor representation of rotations is not minimal. Therefore, one has to deal with a number of degrees of freedom greater than what would be strictly necessary. On the other hand, a representation based on Euler-like angles* allows the use of only three variables to describe rotations. Despite that, also representations based on Euler-like angles involve some issues that might be complex to address, especially in the case of dynamical problems. In particular, they suffer from the so called gimbal lock (Brezov et al. 2013). Also, it is difficult to combine two rotations, as well as Euler-like angels do not vary continuously along arbitrarily large motions (Pai 2011).

An alternative way to describe rotations is the angle/axis representation. However, in this case, there are disadvantages too. Apart from being a non-minimal representation (four degrees of freedom instead of three), such a representation is also singular when no rotation is considered. Besides, there might be a lack of continuity along the motion, and not be possible to combine conveniently several rotations.

A possible solution to the issues mentioned above is the use of quaternions. They are a generalization of complex numbers that involves no trigonometric functions and are characterized by four scalar parameters. Therefore this encoding is not minimal. In fact, a quaternion represents a rotation only if the constraint of unitary amplitude is considered. As the orientation changes, the quaternion elements vary continuously over the unit sphere in $\mathbb{R}^{4}$, and it is convenient to combine two or more rotations using the quaternion product. Nevertheless, this representation is not unique. Indeed quaternions with opposite sign represent the same rotation. To sum up, this representation is quite complicated, and it is often troublesome to employ the four parameters required by such encoding directly, without using the matrix representation of the rotation.

In this paper, a novel discrete formulation is proposed which specifies the orientation of the cross-sections of the rod utilizing a set of auxiliary points. These last define the material directions of the principal axes of the cross-sections. The rationale behind this approach is to overcome the difficulties previously mentioned, and especially the lack of continuity along the motion path, without resorting to the complicated quaternion representation.

The problem here presented is set in a discrete framework by following the Hencky bar-chain approach (Turco et al. 2016; Wang et al. 2015; Zhang et al. 2016a,b). Although the great majority of literature concern the planar case, there are works generalizing the Hencky model also for three-dimensional motions (Turco 2018). Therefore, following the general idea employed in Turco (2018) and generalizing the formulation presented in Baroudi et al. (2019), an articulated system of bars is considered herein, each experiencing only stretching deformation. In particular, elastic lumped joints connect

\footnotetext{
*It is possible to define different sets of Euler angles. Actually there exist twelve of them, depending on the particular adopted sequence of rotation axes.
} 
adjacent segments. The definition of the elastic behavior of the connections is closely related with the three discrete curvatures describing the change in the orientation of two consecutive material triads of unit vectors, which define the positioning of cross-sections of the beam and are rigidly bonded on the corresponding segment in the articulated chain of bars.

\section{An elastic model of rod: geometrical exact formulation}

Following the hypotheses of Kirchhoff and Clebsch (Coleman et al. 1993), an elastic rod characterized by stretching, bending, and twisting deformations is considered. This onedimensional system is kinematically represented by its centerline and by a vector field of orthogonal triads describing the orientation of the cross-sections of the elastic rod. Moreover, in the case of a very slender rod (i.e., when the diameter ${ }^{\dagger}$ of cross-sections is very small compared with its length), it is commonly accepted to assume that one vector of the triad is parallel to the tangent to the centerline (i.e., shear deformation negligible). In these circumstances, the kinematics of the $\operatorname{rod} \mathscr{B}$, in the framework of a material formulation, can be defined by the following map

$$
\chi: \mathscr{B} \times \mathbb{R} \rightarrow \mathscr{E} \times \mathcal{V}
$$

where $\mathscr{E}$ is the three-dimensional Euclidean space and $\mathcal{V}$ is the translational space of $\mathscr{E}$, and more specifically

$$
\chi:(S, t) \mapsto(\boldsymbol{x}, \boldsymbol{v})
$$

according to which each point of the rod labeled by $S \in[0, L]$, i.e., the abscissa along the centerline of length $L$, and any given time $t \in \mathbb{R}$ are mapped into a place $\boldsymbol{x} \in \mathscr{E}$ which describes the current position of the centerline and a vector $\boldsymbol{v} \in \mathcal{V}$ which represents the orientation of one principal axis of the cross-section. The vector $\boldsymbol{v}$ should be such that its length is constant $\left(\|v\|=\mathrm{d}_{1}\right)$ and it remains orthogonal to the tangent to the centerline during all the motion $\left(\boldsymbol{v} \cdot \boldsymbol{x}^{\prime}=0^{\ddagger}\right)$. Of course, the other principal axis of the cross-section is automatically given in the considered hypotheses, because it is orthogonal to $\boldsymbol{x}^{\prime}$ and $\boldsymbol{v}$. In other words, the vector $\boldsymbol{v}$ defines a specific point, not necessarily a material point, on the principal axis considered, i.e.

$$
\boldsymbol{y}=\boldsymbol{x}+\boldsymbol{v}
$$

With these definitions, the kinematics of the rod can be fully described by the two fields

$$
(\boldsymbol{x}(S, t), \boldsymbol{y}(S, t))
$$

which must be complemented by the following constraints

$$
\begin{aligned}
\|\boldsymbol{y}-\boldsymbol{x}\| & =\mathrm{d}_{1} \\
(\boldsymbol{y}-\boldsymbol{x}) \cdot \boldsymbol{x}^{\prime} & =0
\end{aligned}
$$

\footnotetext{
'Here 'diameter' means the maximum among all distances between pairs of points in the cross-section.

$\ddagger$ The prime denotes differentiation with respect to $S$.
} 
Naturally, one may solve these constrained equations (5) and proceed by the usual way, reducing the introduced extra-variables. However, given the nonlinearity of these equations, the problem could be tough to handle. The method of Lagrange multipliers provides an alternative and convenient way to manage this matter. In the case of continuous bodies, particular care should be taken, though. Indeed, the anew introduced variables, i.e. the Lagrange multipliers, must belong to a proper functional space endowed with a suitable inner product. Such a functional space should be defined consistently with the energy functional into play to describe the behavior of the system (see for more details Bersani et al. (2019); dell'Isola and Di Cosmo (2018)).

Using the variables $\boldsymbol{x}$ and $\boldsymbol{y}$, it is easy to evaluate the unit vectors of the triads which assign the orientation of the cross-sections of the rod

$$
\boldsymbol{e}=\left\|\frac{\partial \boldsymbol{x}}{\partial S}\right\|^{-1} \frac{\partial \boldsymbol{x}}{\partial S}, \quad \boldsymbol{m}=\frac{\boldsymbol{y}-\boldsymbol{x}}{\|\boldsymbol{y}-\boldsymbol{x}\|}, \quad \boldsymbol{n}=\boldsymbol{e} \times \boldsymbol{m}
$$

where $\boldsymbol{e}$ is the unit tangent vector to the centerline, and $\boldsymbol{m}$ and $\boldsymbol{n}$ are the two directions of the principal axes of the cross-sections.

Among the placements $\chi$, one of them is selected as a reference configuration and introducing the notation $(\boldsymbol{X}(S), \boldsymbol{Y}(S))$ for it being $S$ the natural parameter of $\boldsymbol{X}$, the reference directors $(\boldsymbol{E}(S), \boldsymbol{M}(S), \boldsymbol{N}(S))$ can be also evaluated using the relations (6).

The strain measure along the axial direction $\varepsilon(S, t)$ is defined as

$$
\varepsilon=\left\|\frac{\partial \boldsymbol{x}}{\partial S}\right\|-\left\|\frac{\partial \boldsymbol{X}}{\partial S}\right\|=\left\|\frac{\partial \boldsymbol{x}}{\partial S}\right\|-1
$$

The unit vectors of the triads define the rotation tensor $R$

$$
R:\left(\mathbf{D}_{1}, \mathbf{D}_{2}, \mathbf{D}_{3}\right) \mapsto(\boldsymbol{e}, \boldsymbol{m}, \boldsymbol{n})
$$

which maps the unit vectors of the observational frame of reference $\mathfrak{R}\left(\mathcal{O}, \mathbf{D}_{1}, \mathbf{D}_{2}, \mathbf{D}_{3}\right)$ into the directors which represent the current orientation of the cross-sections. Therefore, the second order curvature tensor $W$ expressed in terms of rotations is

$$
W=R^{\top} R^{\prime}
$$

from which it is possible to evaluate the curvatures related to the change of orientation of the triad $(\boldsymbol{e}, \boldsymbol{m}, \boldsymbol{n})$ along the centerline as the abscissa $S$ varies in the following way:

$$
k_{1}=-\frac{\partial \boldsymbol{n}}{\partial S} \cdot \boldsymbol{m}, \quad k_{2}=\frac{\partial \boldsymbol{e}}{\partial S} \cdot \boldsymbol{n}, \quad k_{3}=-\frac{\partial \boldsymbol{m}}{\partial S} \cdot \boldsymbol{e}
$$

Although the curvatures (10) are appropriate for an ample amount of applications, in some cases, when considerable stretching is involved (dell'Isola et al. 2016, 2019), it is preferable a definition for them, which takes into account the current elongation. Therefore, keeping in mind those applications, a slightly different definition of the 
curvatures is introduced to take into account the change of length of the rod. In formulae

$$
\kappa_{1}=-\left\|\frac{\partial \boldsymbol{x}}{\partial S}\right\| \frac{\partial \boldsymbol{n}}{\partial S} \cdot \boldsymbol{m}, \quad \kappa_{2}=\left\|\frac{\partial \boldsymbol{x}}{\partial S}\right\| \frac{\partial \boldsymbol{e}}{\partial S} \cdot \boldsymbol{n}, \quad \kappa_{3}=-\left\|\frac{\partial \boldsymbol{x}}{\partial S}\right\| \frac{\partial \boldsymbol{m}}{\partial S} \cdot \boldsymbol{e}
$$

The same expressions are considered for the references curvatures

$$
K_{1}=-\frac{\partial \boldsymbol{N}}{\partial S} \cdot \boldsymbol{M}, \quad K_{2}=\frac{\partial \boldsymbol{E}}{\partial S} \cdot \boldsymbol{N}, \quad K_{3}=-\frac{\partial \boldsymbol{M}}{\partial S} \cdot \boldsymbol{E}
$$

From the above definitions, the measures related to torsion $(\tau)$ and the bending with respect to the directions $\boldsymbol{m}\left(\beta_{2}\right)$ and $\boldsymbol{n}\left(\beta_{3}\right)$ become

$$
\tau=\kappa_{1}-K_{1}, \quad \beta_{2}=\kappa_{2}-K_{2}, \quad \beta_{3}=\kappa_{3}-K_{3}
$$

The strain energy density is a convex function of the strain measures above-mentioned

$$
\Psi=\Psi\left(\varepsilon, \tau, \beta_{2}, \beta_{3}\right)
$$

Finally, denoting with dot the differentiation with respect to the time, the material angular velocity can be expressed by a second order skew tensor

$$
\Omega=R^{\top} \dot{R}
$$

or equivalently by the components of the axial vector of $\Omega$, i.e. ${ }^{\S}$

$$
\omega_{1}=-\frac{\partial \boldsymbol{n}}{\partial t} \cdot \boldsymbol{m}=\boldsymbol{\omega} \cdot \boldsymbol{e}, \quad \omega_{2}=\frac{\partial \boldsymbol{n}}{\partial t} \cdot \boldsymbol{e}=\boldsymbol{\omega} \cdot \boldsymbol{m}, \quad \omega_{3}=-\frac{\partial \boldsymbol{m}}{\partial t} \cdot \boldsymbol{e}=\boldsymbol{\omega} \cdot \boldsymbol{n}
$$

where $\boldsymbol{\omega}$ is the angular velocity vector of the triad referred to the observational frame of reference $\mathfrak{R}$. Thus, the kinetic energy density is a function of the velocity of the points of mass centers laying on the centerline and the angular velocity of the cross-sections assumed as rigid bodies, i.e.

$$
\mathscr{K}(\dot{\boldsymbol{x}}, \boldsymbol{\omega})=\frac{1}{2} \int_{0}^{L}\left[\varrho \dot{\boldsymbol{x}} \cdot \dot{\boldsymbol{x}}+a(\boldsymbol{\omega} \cdot \boldsymbol{e})^{2}+b(\boldsymbol{\omega} \cdot \boldsymbol{m})^{2}+c(\boldsymbol{\omega} \cdot \boldsymbol{n})^{2}\right] \mathrm{d} S
$$

whose explicit form is obtained applying König's theorem. The material parameters are the mass density per unit line, $\varrho$, and the moments of inertia, $a, b$, and $c$ per unit line around the current axes, $\boldsymbol{e}, \boldsymbol{m}$, and $\boldsymbol{n}$, respectively.

\section{The discrete formulation}

To discretize the map $\chi$ of Eq. (1), the two sequences $\left(\boldsymbol{x}_{n}\right)_{n \in\left\{1,2, \ldots N_{e}\right\}}$ and $\left(\boldsymbol{y}_{n}\right)_{n \in\left\{1,2, \ldots N_{e}\right\}}$ of finite length $N_{e}$ are introduced, associating to them the discrete

\footnotetext{
${ }^{\S}$ Here the Poisson's formulae have been used: $\dot{n}=\boldsymbol{\omega} \times \boldsymbol{n}, \dot{\boldsymbol{m}}=\boldsymbol{\omega} \times \boldsymbol{m}$
} 


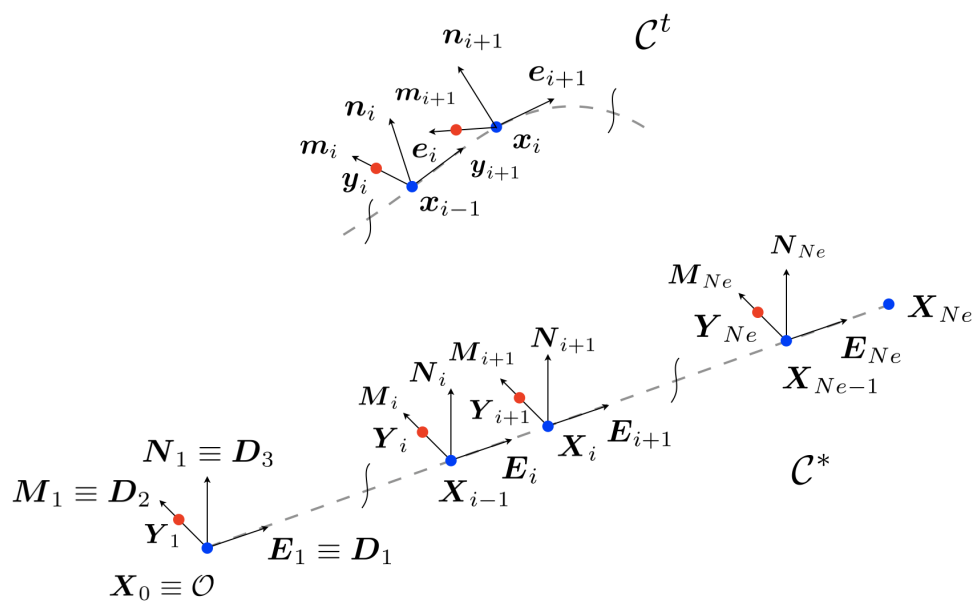

Figure 1. Kinematical description of a discrete rod: reference, $\mathcal{C}^{*}$, and current, $\mathcal{C}^{t}$, configuration.

sequence $S_{n}$ in which the abscissa $S$ has been sampled, as follows

$$
\boldsymbol{x}_{n}(t)=\boldsymbol{x}\left(S_{n}, t\right), \quad \boldsymbol{y}_{n}(t)=\boldsymbol{y}\left(S_{n-1}, t\right)
$$

They describe the current positions of $N_{e}$ points on the centerline of the rod and the points taken on the principal axis of the cross-sections. For the sake of simplicity, a uniform discretization $S_{n}-S_{n-1}=\eta$ is assumed $\forall n \in\left\{1,2, \ldots N_{e}\right\}$, where $S_{0}$ and $S_{N_{e}}$ are 0 and $L$, respectively. Moreover, the initial point of the sequence of the centerline $\boldsymbol{x}_{0}$ is identified with its location in the reference configuration $\boldsymbol{X}_{0}$ and simultaneously with the origin of the observational frame of reference $\mathcal{O}$, i.e., $\boldsymbol{x}_{0}=\boldsymbol{X}_{0}=\mathcal{O}$ (see Fig. 1).

In a discrete context, the unit vectors of the triads related to the kinematics of the rod are evaluated as

$$
\boldsymbol{e}_{i}(t)=\frac{\boldsymbol{x}_{i}(t)-\boldsymbol{x}_{i-1}(t)}{\left\|\boldsymbol{x}_{i}(t)-\boldsymbol{x}_{i-1}(t)\right\|}, \quad \boldsymbol{m}_{i}(t)=\frac{\boldsymbol{y}_{i}(t)-\boldsymbol{x}_{i-1}(t)}{\left\|\boldsymbol{y}_{i}(t)-\boldsymbol{x}_{i-1}(t)\right\|}, \quad \boldsymbol{n}_{i}(t)=\boldsymbol{e}_{i}(t) \times \boldsymbol{m}_{i}(t)
$$

The analogous expressions for the positions $\left(\boldsymbol{X}_{i}, \boldsymbol{Y}_{i}\right)$ and the unit vectors $\left(\boldsymbol{E}_{i}, \boldsymbol{M}_{i}, \boldsymbol{N}_{i}\right)$ in the reference configuration should be taken into consideration as well.

The constraints (5), once the necessary changes for the discrete formulation have been made, become

$$
\begin{aligned}
& G_{1 i}\left(\boldsymbol{x}_{i}, \boldsymbol{y}_{i}\right)=\left\|\boldsymbol{y}_{i}(t)-\boldsymbol{x}_{i-1}(t)\right\|-\mathrm{d}_{1}=0 \\
& G_{2 i}\left(\boldsymbol{x}_{i}, \boldsymbol{y}_{i}\right)=\boldsymbol{e}_{i}(t) \cdot \boldsymbol{m}_{i}(t)=0
\end{aligned}
$$

for $i \in\left\{1,2, \ldots N_{e}\right\}$. 
The discrete counterpart of the strain measure along the axial direction (7) is

$$
\varepsilon_{i}(t)=\left\|\boldsymbol{x}_{i}(t)-\boldsymbol{x}_{i-1}(t)\right\|-\eta
$$

as a matter of fact, is the difference between the current distance of two adjacent points and the corresponding one into the reference configuration.

The curvatures associated to the relative positions of two adjacent triads are evaluated by

$$
\begin{aligned}
& \kappa_{1 i}(t)=-\frac{1}{\eta}\left\|\boldsymbol{x}_{i}(t)-\boldsymbol{x}_{i-1}(t)\right\|\left\{\frac{1}{\eta}\left[\boldsymbol{n}_{i}(t)-\boldsymbol{n}_{i-1}(t)\right] \cdot \boldsymbol{m}_{i-1}(t)\right\} \\
& \kappa_{2 i}(t)=\frac{1}{\eta}\left\|\boldsymbol{x}_{i}(t)-\boldsymbol{x}_{i-1}(t)\right\|\left\{\frac{1}{\eta}\left[\boldsymbol{e}_{i}(t)-\boldsymbol{e}_{i-1}(t)\right] \cdot \boldsymbol{n}_{i-1}(t)\right\} \\
& \kappa_{3 i}(t)=-\frac{1}{\eta}\left\|\boldsymbol{x}_{i}(t)-\boldsymbol{x}_{i-1}(t)\right\|\left\{\frac{1}{\eta}\left[\boldsymbol{m}_{i}(t)-\boldsymbol{m}_{i-1}(t)\right] \cdot \boldsymbol{e}_{i-1}(t)\right\}
\end{aligned}
$$

in accordance with the Eqs. (11), for $i \in\left\{2, \ldots N_{e}\right\}$, while for $i=1$ they are specified by

$$
\begin{aligned}
& \kappa_{11}(t)=-\frac{1}{\eta}\left\|\boldsymbol{x}_{i}(t)\right\|\left\{\frac{1}{\eta}\left[\boldsymbol{n}_{1}(t)-\mathbf{D}_{3}\right] \cdot \mathbf{D}_{2}\right\} \\
& \kappa_{21}(t)=\frac{1}{\eta}\left\|\boldsymbol{x}_{i}(t)\right\|\left\{\frac{1}{\eta}\left[\boldsymbol{e}_{1}(t)-\mathbf{D}_{1}\right] \cdot \mathbf{D}_{3}\right\} \\
& \kappa_{31}(t)=-\frac{1}{\eta}\left\|\boldsymbol{x}_{i}(t)\right\|\left\{\frac{1}{\eta}\left[\boldsymbol{m}_{1}(t)-\mathbf{D}_{2}\right] \cdot \mathbf{D}_{1}\right\}
\end{aligned}
$$

Plainly, one can evaluate the differentiation with a higher order of accuracy, but this is beyond the purpose of the paper; thus, this kind of calculation is omitted.

Therefore, since the curvatures are the derivatives of the relative angular distortions between cross-sections, these last angles can be evaluated by numerically integrating the curvatures with the same order of the accuracy of before, in the following way

$$
\vartheta_{i}(t)=\eta \kappa_{1 i}(t), \quad \varphi_{2 i}(t)=\eta \kappa_{2 i}(t), \quad \varphi_{3 i}(t)=\eta \kappa_{3 i}(t)
$$

Figure 2 provides a simple explanation of the geometrical meaning of the relative angular distortions for the elemental cases of pure rotations made with respect to the current unit vectors (highlighted in dark red) without taking into account any extension of the rod.

Of course, the same is valid for the reference configuration; thus one obtains

$$
\begin{aligned}
& K_{1 i}=-\frac{1}{\eta}\left[\boldsymbol{N}_{i}-\boldsymbol{N}_{i-1}\right] \cdot \boldsymbol{M}_{i-1} \\
& K_{2 i}=\frac{1}{\eta}\left[\boldsymbol{E}_{i}-\boldsymbol{E}_{i-1}\right] \cdot \boldsymbol{N}_{i-1} \\
& K_{3 i}=-\frac{1}{\eta}\left[\boldsymbol{M}_{i}-\boldsymbol{M}_{i-1}\right] \cdot \boldsymbol{E}_{i-1}
\end{aligned}
$$




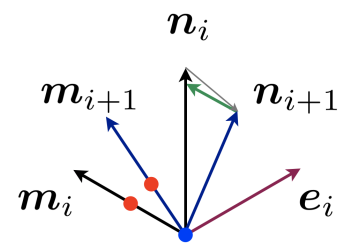

(a) $\vartheta_{i+1}$
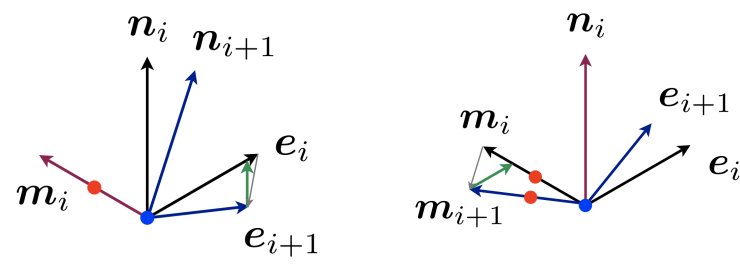

Figure 2. Discrete definition of the relative angular distortions.

for $i \in\left\{2, \ldots N_{e}\right\}$ and

$$
\begin{aligned}
& K_{11}=-\frac{1}{\eta}\left[\boldsymbol{N}_{1}-\mathbf{D}_{3}\right] \cdot \mathbf{D}_{2} \\
& K_{21}=\frac{1}{\eta}\left[\boldsymbol{E}_{1}-\mathbf{D}_{1}\right] \cdot \mathbf{D}_{3} \\
& K_{31}=-\frac{1}{\eta}\left[\boldsymbol{M}_{1}-\mathbf{D}_{2}\right] \cdot \mathbf{D}_{1}
\end{aligned}
$$

for $i=1$. The reference angular distortions are

$$
\Theta_{i}=\eta K_{1 i}, \quad \Phi_{2 i}=\eta K_{2 i}, \quad \Phi_{3 i}=\eta K_{3 i}
$$

Eventually, the measures of deformations related to torsion, and bending along the axes $\boldsymbol{m}_{i}$ and $\boldsymbol{n}_{i}$ are

$$
\tau_{i}(t)=\vartheta_{i}(t)-\Theta_{i}, \quad \beta_{2 i}(t)=\varphi_{2 i}(t)-\Phi_{2 i}, \quad \beta_{3 i}(t)=\varphi_{3 i}(t)-\Phi_{3 i}
$$

respectively.

The elastic energy is

$$
\Psi^{\mathrm{el}}=\frac{1}{2} \sum_{i=1}^{N_{e}}\left[A_{i}\left(\varepsilon_{i}\right)^{2}+B_{i}\left(\tau_{i}\right)^{2}+C_{i}\left(\beta_{2 i}\right)^{2}+D_{i}\left(\beta_{3 i}\right)^{2}\right]
$$

where the coefficients $A_{i}, B_{i}, C_{i}, D_{i}$ are positive constants given by

$$
A_{i}=\frac{Y_{\mathrm{m}} \mathcal{A}_{\mathrm{cs} i}}{\eta}, \quad B_{i}=\frac{G_{\mathrm{m}} J_{\mathrm{p}_{i}}}{\eta q_{\mathrm{t} i}}, \quad C_{i}=\frac{Y_{\mathrm{m}} J_{\boldsymbol{m}_{i}}}{\eta}, \quad D_{i}=\frac{Y_{\mathrm{m}} J_{\boldsymbol{n}_{i}}}{\eta}
$$

for $i \in\left\{2, \ldots N_{e}\right\} . Y_{\mathrm{m}}$ and $G_{\mathrm{m}}$ are the Young and the shear moduli of the material of the rod, respectively. $\mathcal{A}_{\mathrm{cs} i}$ is the area of the cross-section, $J_{\mathrm{p}_{i}}, J_{\boldsymbol{m}_{i}}$ and $J_{\boldsymbol{n}_{i}}$ are the torsional, and the flexural inertial moments related to the directions $\boldsymbol{m}_{i}$ and $\boldsymbol{n}_{i}$ of the cross-section, 
respectively. The quantity $q_{\mathrm{t}_{i}}$ is the shear factor of the cross-section. Its values for $i=1$ define the stiffnesses of the constrains that link the rod to the ground. In the case of a clamp, it is possible to set

$$
A_{1}=2 \frac{Y_{\mathrm{m}} \mathcal{A}_{\mathrm{cs} 1}}{\eta}, \quad B_{1}=2 \frac{G_{\mathrm{m}} J_{\mathrm{p}_{1}}}{\eta q_{\mathrm{t} 1}}, \quad C_{1}=2 \frac{Y_{\mathrm{m}} J_{\boldsymbol{m}_{1}}}{\eta}, \quad D_{1}=2 \frac{Y_{\mathrm{m}} J_{\boldsymbol{n}_{1}}}{\eta}
$$

The rationale behind this assumption can be explained considering to evaluate the lumped stiffnesses in each fictitious discrete joint $\boldsymbol{X}_{i}$ as related to half part of the previous segment between the cross-sections $S_{i-1}$ and $S_{i}$ and half part to the following one between $S_{i}$ and $S_{i+1}$. Therefore, for the interior points, this concentrated stiffness results from an arch-length equal to the length of an entire segment, $\eta$. Diversely the stiffnesses related to the end parts of the entire rod correspond to half part of the segment, thus $\eta / 2$. In the case of a hinge, $C_{1}$ or $D_{1}$ is zero depending on how the hinge axis is arranged. Indeed, the rod does not perceive any bending moment respect to the axis of the hinge.

Since the rod under consideration is very compliant, the external action due to the weight is not negligible at all. Therefore, the gravitational potential must be introduced and is

$$
\Psi^{\mathrm{wg}}=\sum_{i=1}^{N_{e}} g m_{i} \boldsymbol{x}_{i} \cdot \mathbf{D}_{3}
$$

where the mass coefficients are given by $m_{i}=m_{\mathrm{tot}} / N_{e}$ for $i \in\left\{1,2, \ldots N_{e}-1\right\}$ and $m_{N e}=m_{\mathrm{tot}} /\left(2 N_{e}\right)$ in terms of the total mass of the rod, $m_{\mathrm{tot}}$, for a uniform distribution of the mass. In other words, the mass of a discrete piece of beam between two adjacent points $\boldsymbol{x}_{i-1}$ and $\boldsymbol{x}_{i}$ is divided into two and placed at the same points. In the case examined, this assumption is equivalent to put a mass of $m_{\mathrm{tot}} / N_{e}$ in the locations of mass centers of each finite slab of the rod within the abscissae $S_{i-1}$ and $S_{i}$.

If each finite segment of the rod with uniform distribution of the mass within the abscissae $S_{i-1}$ and $S_{i}$ is assumed to be a rigid body, as in the original formulation of Hencky, the kinetic energy becomes

$$
\mathscr{K}=\frac{1}{2} \sum_{i=1}^{N_{e}}\left[m_{i} \dot{\boldsymbol{x}}_{i} \cdot \dot{\boldsymbol{x}}_{i}+a_{i}\left(\boldsymbol{\omega}_{i} \cdot \boldsymbol{e}_{i}\right)^{2}+b_{i}\left(\boldsymbol{\omega}_{i} \cdot \boldsymbol{m}_{i}\right)^{2}+c_{i}\left(\boldsymbol{\omega}_{i} \cdot \boldsymbol{n}_{i}\right)^{2}\right]
$$

in which $a_{i}, b_{i}$, and $c_{i}$ are the moments of inertia around the principal axes of each slab, i.e., $\boldsymbol{e}_{i}, \boldsymbol{m}_{i}$, and $\boldsymbol{n}_{i}$. Specifically, by making explicit the expressions of the components of the angular velocities as a function of the unit vectors along the principal directions and their time derivatives, the Eq. (41) takes the form

$$
\mathscr{K}=\frac{1}{2} \sum_{i=1}^{N_{e}}\left[m_{i} \dot{\boldsymbol{x}}_{i} \cdot \dot{\boldsymbol{x}}_{i}+a_{i}\left(-\dot{\boldsymbol{n}}_{i} \cdot \boldsymbol{m}_{i}\right)^{2}+b_{i}\left(\dot{\boldsymbol{n}}_{i} \cdot \boldsymbol{e}_{i}\right)^{2}+c_{i}\left(-\dot{\boldsymbol{m}}_{i} \cdot \boldsymbol{e}_{i}\right)^{2}\right]
$$

A little digression about the kinetic energy shall be done to shed light on some important numerical aspects. The kinematical variables used here, namely $\boldsymbol{y}_{i}$, are quite 
efficient in representing the kinetic energy of the cross-sections. However, as far as the elastic energy is concerned, a fascinating choice, in the discrete framework, is to consider the relative rotations between two close triads $\left(\boldsymbol{e}_{i}, \boldsymbol{m}_{i}, \boldsymbol{n}_{i}\right)$ as, e.g., done by using the incremental rotations in Simo (1985); Simo and Vu-Quoc (1986, 1988). Because of the small value of these other variables, all the issues related to the lack of continuity along the motion trajectories lose importance. In spite of this, to evaluate the kinetic energy, the rigid rotation of each cross-section seen from the perspective of the observational frame of reference should be incrementally obtained by multiplying the relative rotation from the beginning of rod to the position of each of the cross-sections. This considerable chain of multiplications involves a computational burden in calculating the kinetic energy which is massive.

The structural dissipation of the rod is modeled by introducing a Rayleigh dissipation function as follows

$$
\mathscr{D}=\frac{1}{2} \sum_{i=1}^{N_{e}}\left[A_{\mathrm{v} i}\left(\dot{\varepsilon}_{i}\right)^{2}+B_{\mathrm{v} i}\left(\dot{\tau}_{i}\right)^{2}+C_{\mathrm{v} i}\left(\dot{\beta}_{2 i}\right)^{2}+D_{\mathrm{v} i}\left(\dot{\beta}_{3 i}\right)^{2}\right]
$$

which depends on the time derivatives of the previously defined measures of deformations (see, e.g., Altenbach and Eremeyev (2015)). The coefficients $A_{\mathrm{v} i}, B_{\mathrm{v} i}$, $C_{\mathrm{v} i}$, and $D_{\mathrm{v} i}$ are positive material parameters (see for the identification of this kind of parameters Lekszycki et al. (1992); Abali et al. (2016)).

For the considered rod, the Lagrangian is assumed to be

$$
\mathscr{L}(\boldsymbol{r}, \dot{\boldsymbol{r}})=\mathscr{K}(\boldsymbol{r}, \dot{\boldsymbol{r}})-\left(\Psi^{\mathrm{el}}(\boldsymbol{r})+\Psi^{\mathrm{wg}}(\boldsymbol{r})\right)
$$

where $\boldsymbol{r}=\left\{\boldsymbol{x}_{1}, \ldots, \boldsymbol{x}_{N e}, \boldsymbol{y}_{1}, \ldots, \boldsymbol{y}_{N e}\right\}$.

By using the method of Lagrange multipliers combined with the generalized Hamilton's principle, the equations of motion are given by

$$
\begin{aligned}
\frac{\mathrm{d}}{\mathrm{d} t}\left(\frac{\partial \mathscr{L}}{\partial \dot{r}_{i}}\right)-\frac{\partial \mathscr{L}}{\partial r_{i}}+\frac{\partial \mathscr{D}}{\partial \dot{r}_{i}} & =\sum_{k=1}^{N_{e}} \lambda_{1 k}(t) \frac{\partial G_{1 k}}{\partial r_{i}}+\sum_{k=1}^{N_{e}} \lambda_{2 k}(t) \frac{\partial G_{2 k}}{\partial r_{i}} & & \text { for } i=1, \ldots 6 N_{e} \\
G_{1 k}\left(r_{1}, \ldots, r_{6 N e}\right) & =0 & & \text { for } k=1, \ldots N_{e} \\
G_{2 k}\left(r_{1}, \ldots, r_{6 N e}\right) & =0 & & \text { for } k=1, \ldots N_{e}
\end{aligned}
$$

together with the initial conditions for the variable $\boldsymbol{r}$ and its derivative $\dot{\boldsymbol{r}}$. Equations (45) are numerically solved by the technical computing system Wolfram Mathematica with a differential-algebraic system of equations (DAEs) solver implemented with the NDSolve routine.

\section{A generalization to an orthotropic rod with Poisson's effect}

The previous formulation lends itself easily to be generalized to an orthotropic rod accounting for Poisson's effect. This kind of generalization is particularly suited for timber or composite beams subject to extensive displacements and rotations. 
The idea, here, is to introduce a new degree of freedom, $d_{2 i}(t)$, as specified below

$$
\boldsymbol{z}_{i}(t)=\boldsymbol{x}_{i-1}(t)+d_{2 i}(t) \boldsymbol{n}_{i}(t)
$$

adding further auxiliary points $\boldsymbol{z}_{i}(t)$ (see fig. 3) as well as to relax the constrained (20a). With these assumptions the further measures of deformations can be defined in this form:

$$
\begin{aligned}
& \varepsilon_{1 i}(t)=\left\|\boldsymbol{y}_{i}(t)-\boldsymbol{x}_{i-1}(t)\right\|-d_{1 i}(t) \\
& \varepsilon_{2 i}(t)=\left\|\boldsymbol{z}_{i}(t)-\boldsymbol{x}_{i-1}(t)\right\|-d_{2 i}(t)
\end{aligned}
$$

to take into account an average macroscopic stretching deformation of the cross-sections along the two principal axes. Thus, the strain energy becomes

$$
\begin{array}{r}
\Psi^{\mathrm{el}}=\frac{1}{2} \sum_{i=1}^{N_{e}}\left[A_{i}\left(\varepsilon_{i}\right)^{2}+A_{1 i}\left(\varepsilon_{1 i}\right)^{2}+A_{2 i}\left(\varepsilon_{2 i}\right)^{2}+H_{1 i} \varepsilon_{i} \varepsilon_{1 i}+H_{2 i} \varepsilon_{i} \varepsilon_{2 i}+\right. \\
\left.B_{i}\left(\tau_{i}\right)^{2}+C_{i}\left(\beta_{2 i}\right)^{2}+D_{i}\left(\beta_{3 i}\right)^{2}\right]
\end{array}
$$

where the coupling terms $\varepsilon_{i} \varepsilon_{1 i}$ and $\varepsilon_{i} \varepsilon_{2 i}$ are responsible for Poisson's effect. The newly introduced material constants $A_{1 i}, A_{2 i}, H_{1 i}$, and $H_{2 i}$ affect the deformation of the crosssection. The constraint (20b) still can be employed with the Lagrange multipliers or substituted with an equivalent term in the energy (48), involving the change of the angle between $\boldsymbol{e}_{i}$ and $\boldsymbol{m}_{i}$ respect to $\pi / 2$, like this: $\frac{1}{2} \sum_{i=1}^{N_{e}} F_{i}\left[\arcsin \left(\boldsymbol{e}_{i} \cdot \boldsymbol{m}_{i}\right)\right]^{2}$.

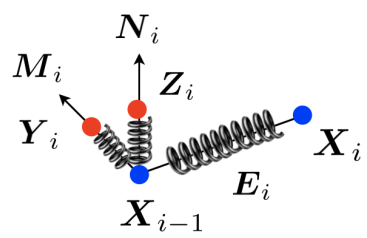

Figure 3. Unit cell for a generalized orthotropic rod with Poisson's effect.

It is worthy to remark that many other kinds of generalization may be done; for example, one can think about generalized beam theory (GBT) (Ferrarotti et al. 2017; Eugster 2015; Piccardo et al. 2014).

\section{Numerical simulations}

\section{Standard static examples}

A preliminary study is carried out to show the level of accuracy of the presented discrete formulation in approximating the corresponding continuous Kirchhoff theory. To this 
purpose, some standard cases are investigated using a soft rod. Herein, with 'soft' rod is intended a beam whose stiffnesses are such that the load due to the own weight involves large deformations and rotations. The sample considered is $0.8 \mathrm{~m}$ long and is characterized by a uniform rectangular cross-section of sizes $(3 \times 0.5) \mathrm{mm}$. The material parameters are the Young modulus, $Y_{\mathrm{m}}$, equal to $78 \mathrm{GPa}$ and the mass density of $2700 \mathrm{~kg} / \mathrm{m}^{3}$. It is worthy specifying that the deformed equilibrium configurations displayed in all figures of this subsection are obtained by a code made with Mathematica solving a constrained minimum problem for the potential energy of the system using the FindMinimum routine.

In the first example, a straight beam clamped at one end, and subjected to a concentrated, counterclockwise moment $M$ at the other end, is considered. It is known that in these circumstances, neglecting the effect of the weight, the equilibrium shapes are circular arcs (see the snapshots in fig. 4). When the end moment is taken to be $M_{0}=2 \pi Y_{\mathrm{m}} J_{m} / L$ the beam bends into a circle. Figure 5 reports a convergence plot in which the size of the elements $\eta$ is plotted versus the tip error, i.e., the norm of the distance between the numerical and the analytical solution of the tip displacement of the cantilever beam for the closed circle subjected to $M_{0}$. The straight trend in the log-log graph is characterized by a slope of 2.036 .

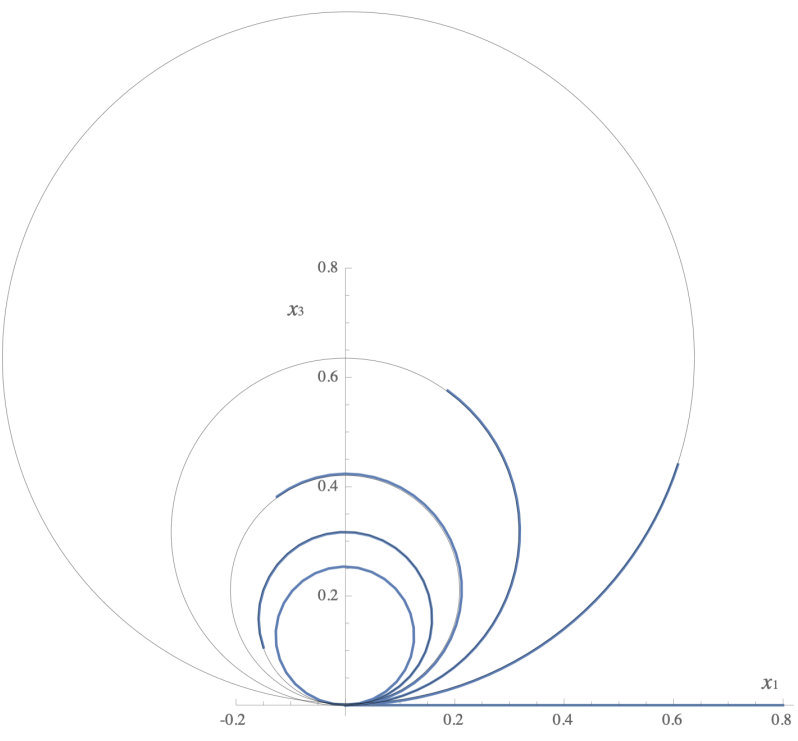

Figure 4. Cantilever beam subjected to an end moment (simulations performed with 30 elements). Configurations for $M=0, M=1 / 5 M_{0}, M=2 / 5 M_{0}, M=3 / 5 M_{0}$, $M=4 / 5 M_{0}$, and $M=M_{0}$.

After this standard benchmark problem, a cantilever beam and a simply-supported beam under the gravity load are taken into account and numerically examined. For 


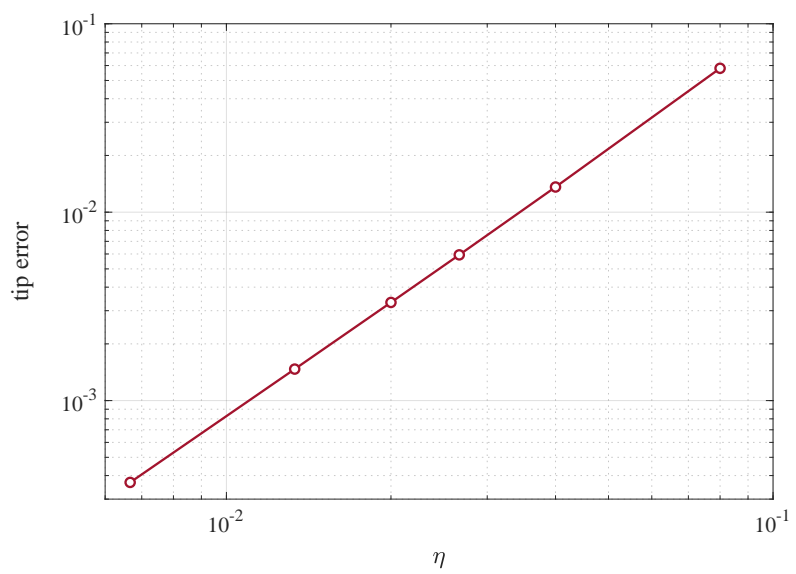

Figure 5. Convergence plot of the cantilever beam subjected to the end moment $M_{0}$.

these two new cases, the equilibrium shapes are evaluated and compared with those obtained with the commercial software Comsol Multiphysics, which allows the study of large deformations and large rotations. For the case of the simply-supported beam the cross-section is set to be $(10 \times 0.2) \mathrm{mm}$. Figures $6 \mathrm{a}$ and $8 \mathrm{a}$ display the comparison between the proposed formulation and the results of Comsol Multiphysics, while Figs. $6 \mathrm{~b}$ and $8 \mathrm{~b}$ indicate the amplitude of the deviation in the equilibrium shapes obtained with the two different approaches. The numerical simulations are performed considering a discretization of the rod with 30 elements. The deviation between the two discrete methods is about 3-4 mm. Indeed, one can achieve the wanted accuracy just considering a proper number of elements. On the other hand, figures 7 and 9 clearly show that the discrete approach proposed reached already an acceptable convergence starting from about 30 elements. This is evident examining both the graphs of the maximum deviation from the configuration prior to the deformation and of the total energy of the rod as the number of elements varies.

\section{Dynamic examples}

The case of biaxial bending with torsion The reference configuration is characterized by a straight centerline of the rod, while the cross-sections are linearly twisted from 0 to $\pi / 4$ in the undeformed state. This particular shape for the rod is selected because all the main kinds of deformation are activated under the gravity load, namely a biaxial bending, i.e., the simultaneous bending about both principal axes of the cross-sections and the torsion. In particular, the case analyzed concerns a rod whose length is $0.8 \mathrm{~m}$ and with a constant rectangular cross-section thick $0.5 \mathrm{~mm}$ and wide $3 \mathrm{~mm}$. The Young 


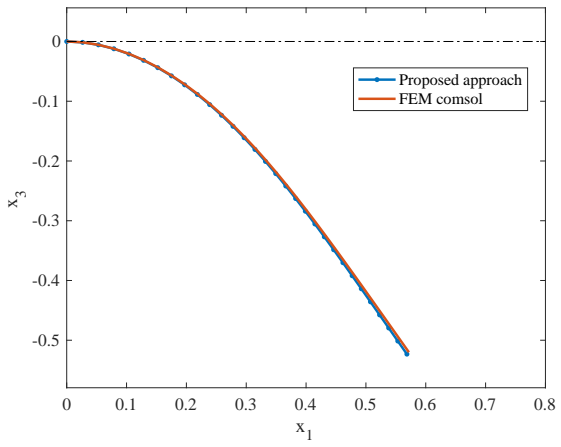

(a) equilibrium shape

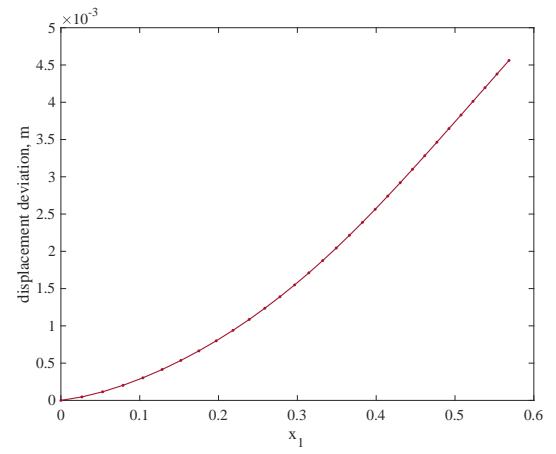

(b) displacement deviation

Figure 6. Case of a cantilever beam: comparison between COMSOL Multiphysics and the discrete formulation presented with 30 elements.

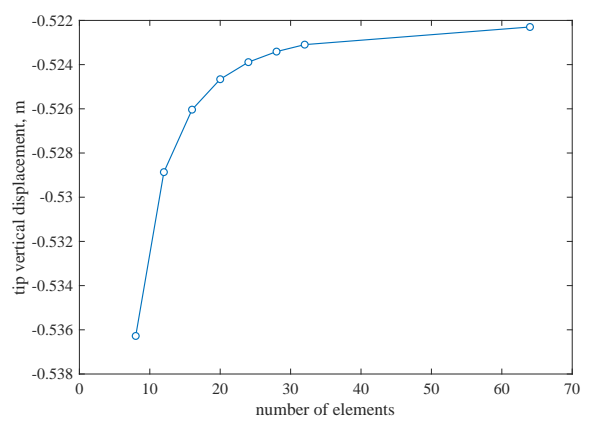

(a) vertical displacement of the tip

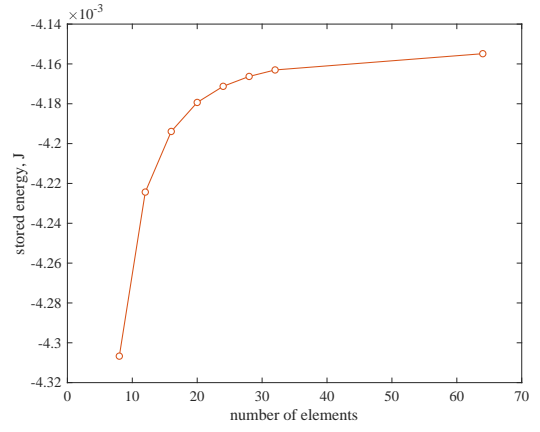

(b) stored energy

Figure 7. Case of a cantilever beam: convergence plots.

modulus is $78 \mathrm{GPa}$, the shear modulus is $30 \mathrm{GPa}$, and the mass density is $2700 \mathrm{~kg} / \mathrm{m}^{3}$. The viscous coefficients are assumed to be $A_{\mathrm{v} i}=2.34 \times 10^{5} \mathrm{~N} \mathrm{~s} / \mathrm{m}, B_{\mathrm{v} i}=0.00168 \mathrm{~N}$ $\mathrm{s} / \mathrm{m}, C_{\mathrm{v} i}=0.00122 \mathrm{~N} \mathrm{~s} / \mathrm{m}$, and $D_{\mathrm{v} i}=0.0439 \mathrm{~N} \mathrm{~s} / \mathrm{m}$. The moments of inertia around the principal axes of each slab are $a_{i}=6.24 \times 10^{-11} \mathrm{~kg} \mathrm{~m}^{2}, b_{i}=2.70 \times 10^{-9} \mathrm{~kg} \mathrm{~m}^{2}$, and $c_{i}=2.76 \times 10^{-9} \mathrm{~kg} \mathrm{~m}^{2}$.

To illustrate the performance of the proposed formulation, the mechanical problem of a cantilever beam, which is accelerated toward the vertical direction $\mathbf{D}_{3}$, is solved with a discretization of 40 elements. Precisely, the initial conditions are the main equilibrium configuration (see Della Corte et al. (2017) for details about other possible equilibrium 


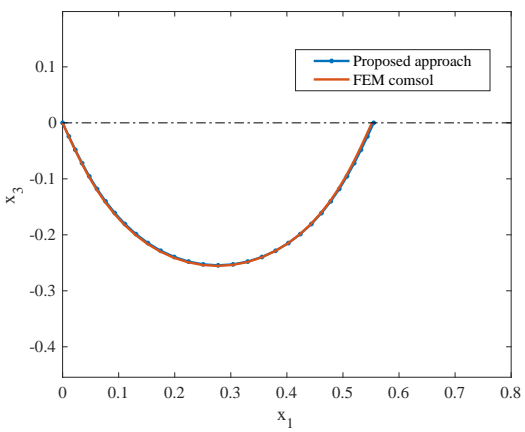

(a) equilibrium shape

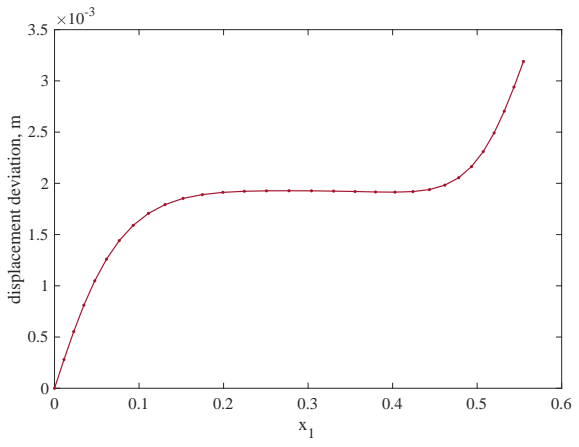

(b) displacement deviation

Figure 8. Case of a simply-supported beam: comparison between COMSOL Multiphysics and the discrete formulation presented with 30 elements.

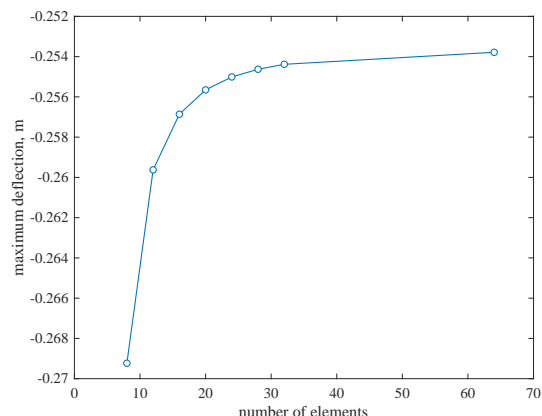

(a) maximum deflection

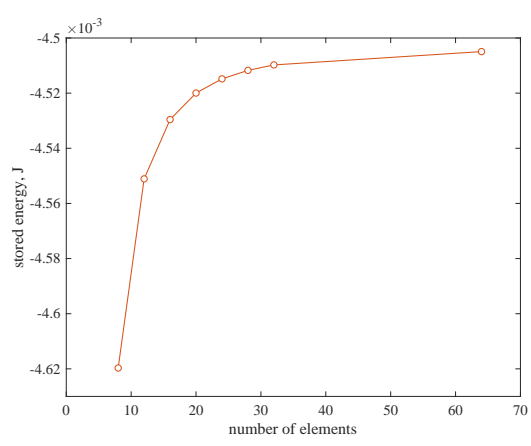

(b) stored energy

Figure 9. Case of a simply-supported beam: convergence plots.

shapes) due to the weight of the rod; and zero velocity. At the clamped left end, the following displacement $y(t)$ is imposed: 


$$
y(t)= \begin{cases}0 & \text { for } t<0 ; \\ A_{y}\left[126\left(\frac{t}{T_{s}}\right)^{5}-420\left(\frac{t}{T_{s}}\right)^{6}+\right. & \\ \left.540\left(\frac{t}{T_{s}}\right)^{7}-315\left(\frac{t}{T_{s}}\right)^{8}+70\left(\frac{t}{T_{s}}\right)^{9}\right] & \text { for } t \in\left[0, T_{s}\right] ; \\ A_{y} & \text { for } t>0 ;\end{cases}
$$

characterized by an amplitude of the seismic oscillation $A_{y}=0.5 \mathrm{~m}$, and a time interval of $T_{s}=0.25 \mathrm{~s}$. This displacement, in the non-inertial reference frame fixed to the left end results in an inertial force which is proportional to the acceleration $\ddot{y}(t)$, shown in fig. 12. In this non-inertial reference frame, as displayed in the following figures, the motion of the beam takes account only of the deformation since, naturally, the prescribed movement to the same reference frame has been filtered apart.

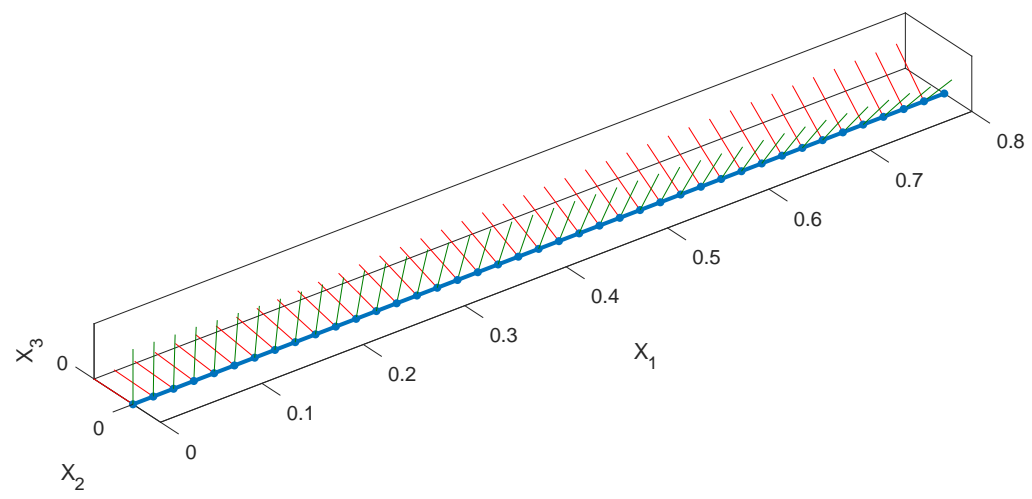

Figure 10. Reference configuration in the undeformed state.

Figures 13 and 14 exhibit the motion of the rod. In fig. 13, the stroboscopic movement on the $x_{1} x_{2}$ and $x_{1} x_{3}$ planes is shown along with the initial configuration, highlighted in red, and an intermediate configuration highly deformed, which is instead underlined with a yellow solid line. Fig. 14 reports the plots of the coordinate of all the points of the centerline as a function of the time. It is worthy to remark that using the Euler angles, it is impossible to describe such a motion simply because the considered trajectory goes beyond the limits that guarantee the continuity of the Euler angels along the motion path.

The nonlinear character of the motion in the initial transient can be easily detected from the fig. 15, where the phase space trajectories of the free extremity coordinates of the rod are plotted. Similarly, the nonlinear effect can be seen from the measures of 

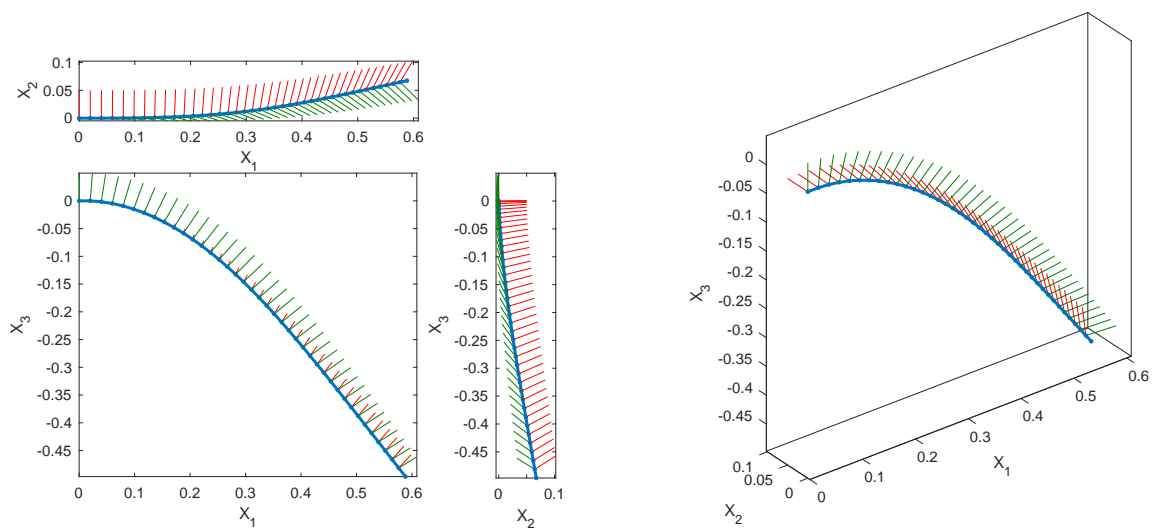

Figure 11. Initial configuration: equilibrium shape under gravity load.

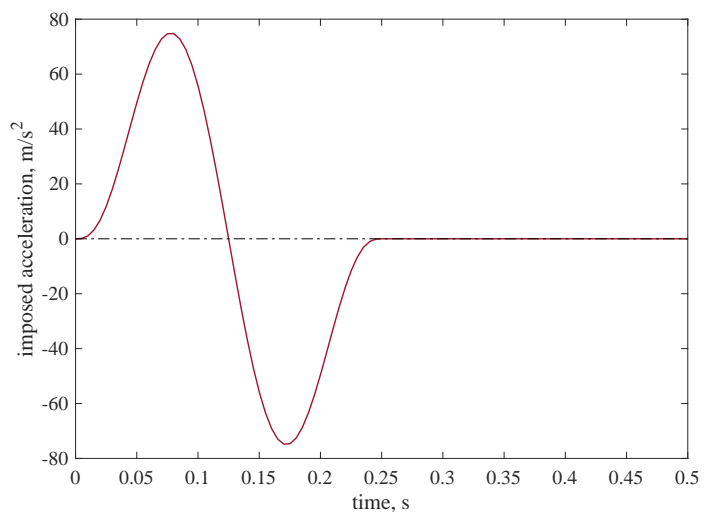

Figure 12. Acceleration due to the motion of the relative frame.

deformations, namely, the torsion $\kappa_{1 i}$ and the curvatures $\kappa_{2 i}$, and $\kappa_{3 i}$ along $\boldsymbol{m}_{i}$ and $\boldsymbol{n}_{i}$ as displayed in fig. 16 at a particular time instant, $0.258 \mathrm{~s}$.

The case of a quick flick of a highly flexible rod from a circular to the equilibrium configuration In this Section, the generalization previously proposed concerning the introduction of Poisson's ratio is tested with an example. A cantilever beam with the same geometry and material constants of the preceding case is the testing sample. Besides the 


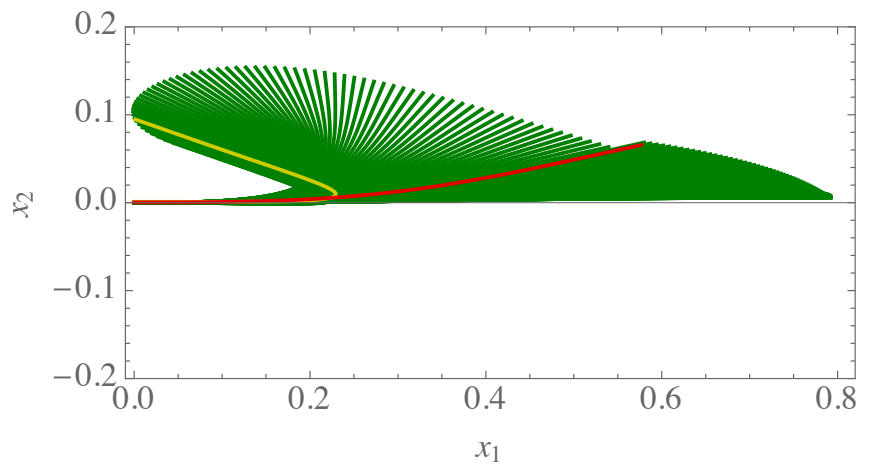

(a) $x_{1} x_{2}$ plane

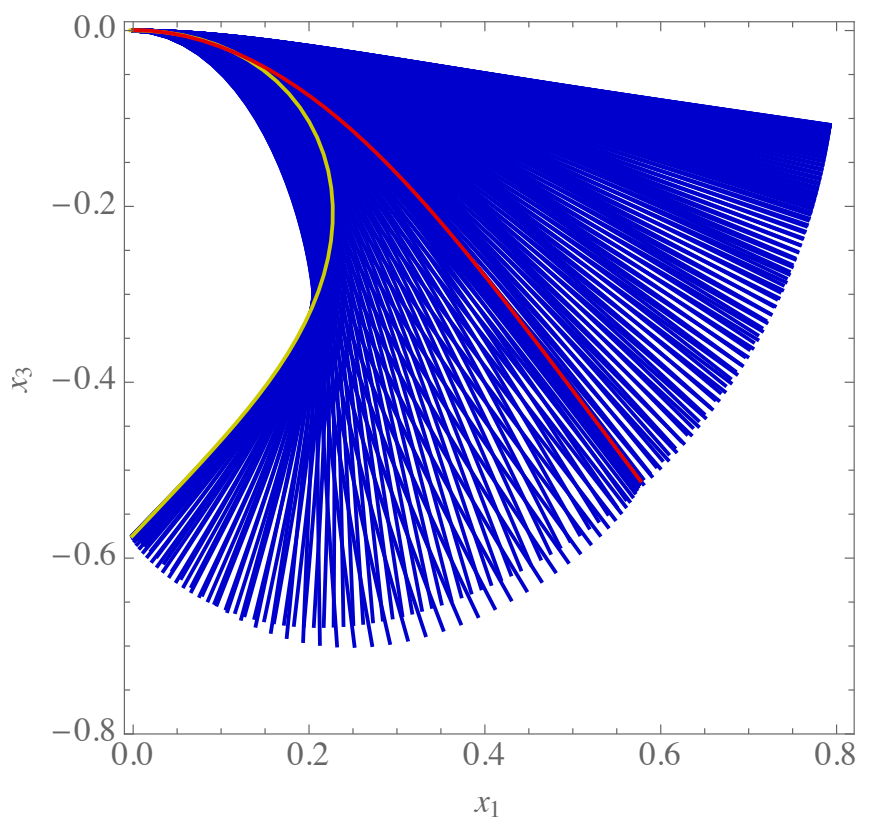

(b) $x_{1} x_{3}$ plane

Figure 13. Stroboscopic motion from 0 to $1.15 \mathrm{~s}$, which correspond approximately to the first largest cycle of the oscillation. The initial configuration is the red solid line. The intermediate configuration at time $0.258 \mathrm{~s}$ is highlighted in yellow.

material constants accountable for the deformation of the cross-sections are assumed to be $A_{1 i}=A_{2 i}=58.5 \mathrm{~N} / \mathrm{m}, H_{1 i}=29.25 \mathrm{~N} / \mathrm{m}$, and $H_{2 i}=20.475 \mathrm{~N} / \mathrm{m}$.

The initial conditions are as far as concerning the shape of the rod centerline, a folded configuration in a circle (see fig. 17a for the directions of the principal axes of cross-sections), and zero velocity $\dot{\boldsymbol{r}}$. Figure 18 shows the temporal evolution of the 

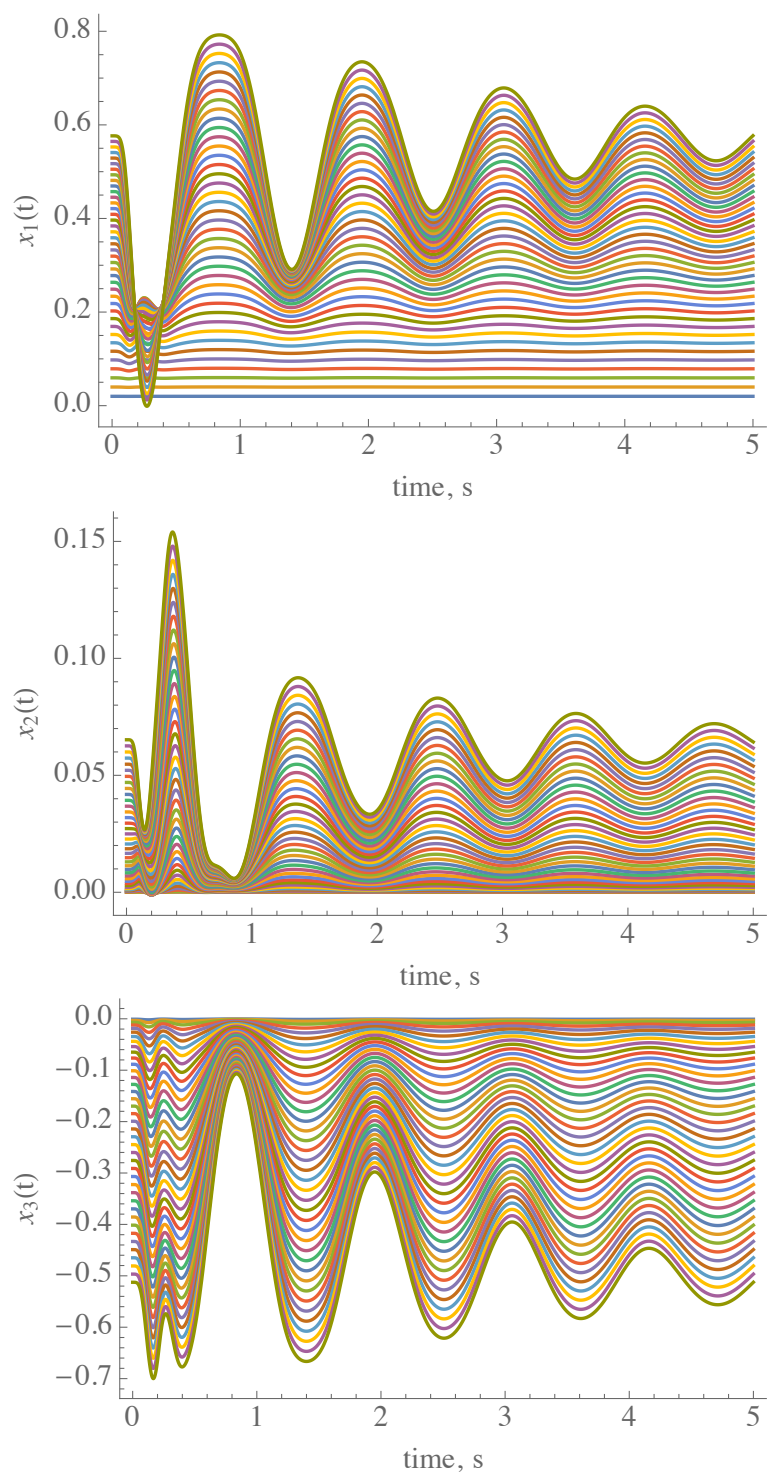

Figure 14. Motion of the rod centerline by coordinates.

significant coordinates of the beam axis line. Figure 19 shows in a single image, with a stroboscopic technique, the progression of the configurations represented in different instants corresponding to equal time intervals from 0 to $0.72 \mathrm{~s}$, namely, the first cycle of oscillation. 


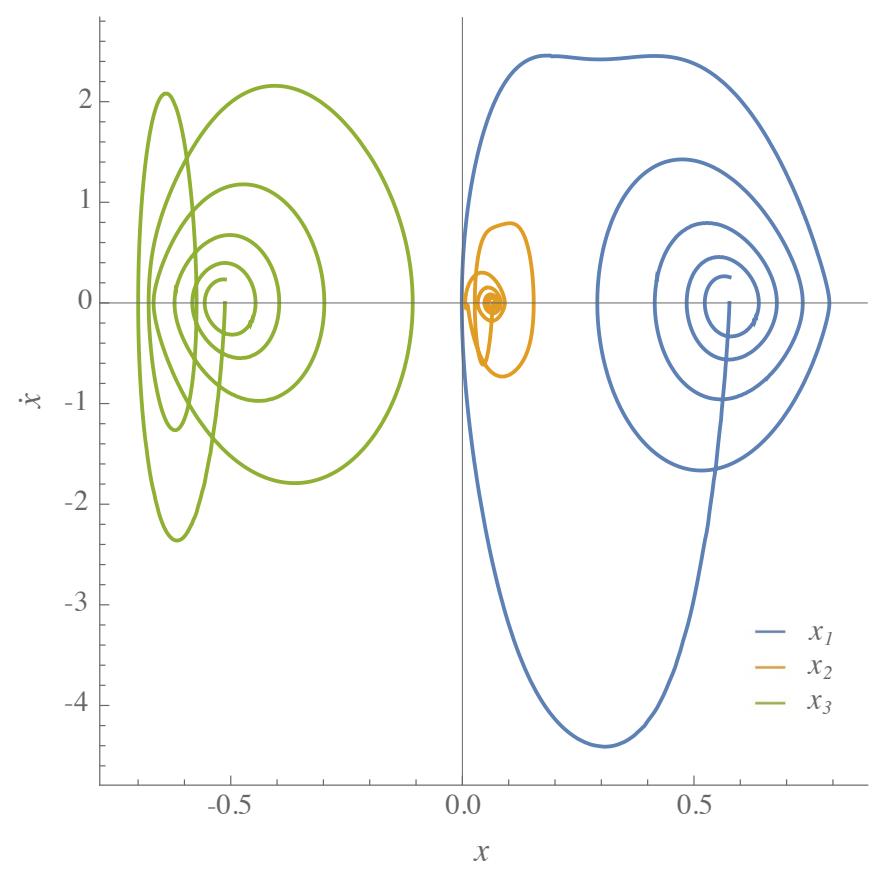

Figure 15. Phase space trajectories of the tip coordinates of the rod.

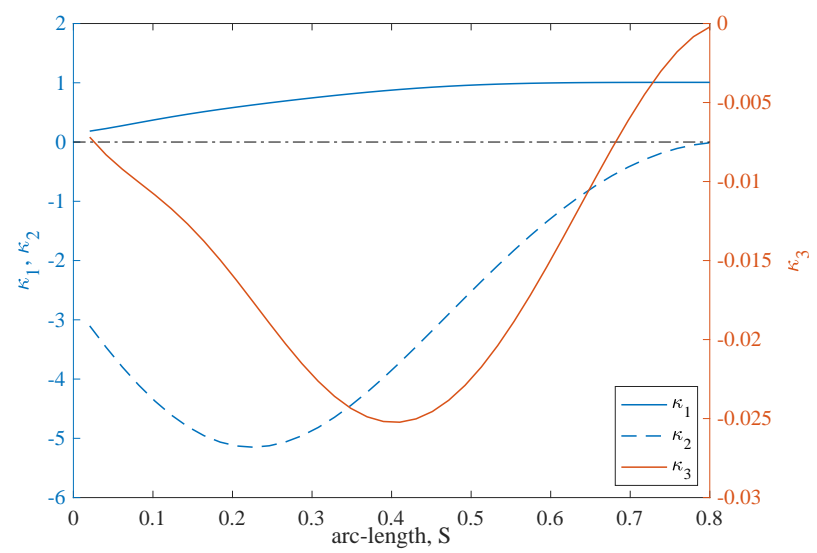

Figure 16. Torsion $\kappa_{1 i}$ and curvatures $\kappa_{2 i}$, and $\kappa_{3 i}$ along $\boldsymbol{m}_{i}$ and $\boldsymbol{n}_{i}$, respectively, at time $0.258 \mathrm{~s}$. 


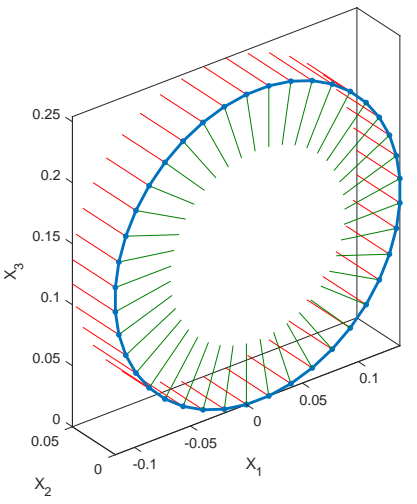

(a) Initial configuration

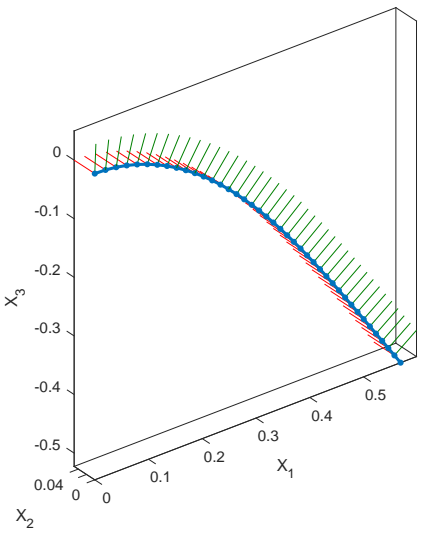

(b) Final configuration

Figure 17. Discrete visualization of the rod at the beginning of the motion and the equilibrium shape toward which the movement tends with 40 elements.

The nonlinear behavior of the motion is also evident in this case if figs. 20, 21, and 22 are observed. Specifically, in figure 20, the intricate and complex trajectories in the phase space of the tip coordinates of the rod are plotted. While figs. 21 and 22 report for two generic instants the measures of deformations of the curvature $\kappa_{2 i}$ along $\boldsymbol{m}_{i}$ and the elongations $\varepsilon_{i}, \varepsilon_{1 i}$ and $\varepsilon_{2 i}$, respectively. Moreover, from fig. 22, Poisson's effect relative to the deformation of the cross-sections is clearly shown.

\section{Conclusions: Present and Future Challenges}

Nowadays, a great deal of research on new materials focuses on so-called metamaterials. They are artificial materials whose macroscopic behavior depends mainly on a microstructure which is designed with the aim of fulfilling a given behavior. Among the panoply of these metamaterials, many of them are characterized by a microstructure made up of small beams arranged in a periodic or quasi-periodic patterns (Barchiesi et al. 2019b; dell'Isola et al. 2015; Avella et al. 1996; Karathanasopoulos et al. 2017; Nejadsadeghi et al. 2019b; Reda et al. 2018; Vangelatos et al. 2019; Spagnuolo et al. 2019). To properly analyze and design these new materials, hence, efficient and easyto-use formulations of beam elements, like the one proposed here, become particularly attractive. In this respect, the pantographic beam model proposed in Alibert et al. (2003); Barchiesi et al. (2019a); Placidi et al. (2020) is owing to its potentialities in real-world applications, quite remarkable. For the sake of completeness, also the one-dimensional architectured materials in Barchiesi and Khakalo (2019); Berezovski et al. (2018); 

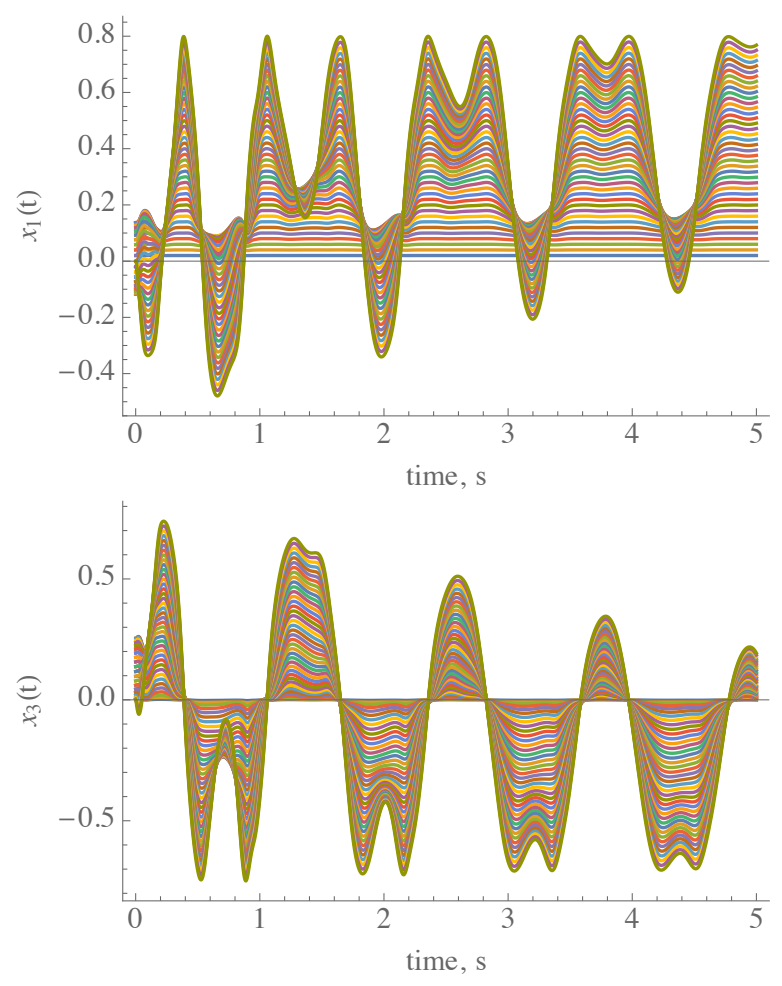

Figure 18. Motion of the rod centerline by coordinates.

Laudato and Barchiesi (2019); Nejadsadeghi et al. (2019a); Niiranen et al. (2019); Solyaev et al. (2019) are worthy of being mentioned.

Again with regard to metamaterials, a very fruitful application of the proposed approach concerns piezo-electro-mechanical beams (Alessandroni et al. 2002; Andreaus et al. 2004; dell'Isola et al. 2003; Lossouarn et al. 2015, 2016). In such 'smart' materials, piezoelectric patches bonded on the host beam are simply a way to draw off mechanical energy towards an electric network. The exchanged energy can then be utilized in different ways: conceiving energy harvesting devices, dissipating the energy into the electric network aiming at damping the vibrations of the mechanical system, etc. It is well-known that the best possible way to exchange energy between the two systems, i.e., mechanical and electrical, is that they share the same behavior, or in other words, the same governing equations. Because of the discrete nature of the equations describing of the electric lumped parameter network, an equivalent discrete formulation for the mechanical system would be obviously very desirable.

Numerical developments in the framework of isogeometric FEM are also enticing, because they allow to obtain even more efficient computing procedures further decreasing the degrees of freedom with the same level of accuracy (see, e.g., for beams in static 


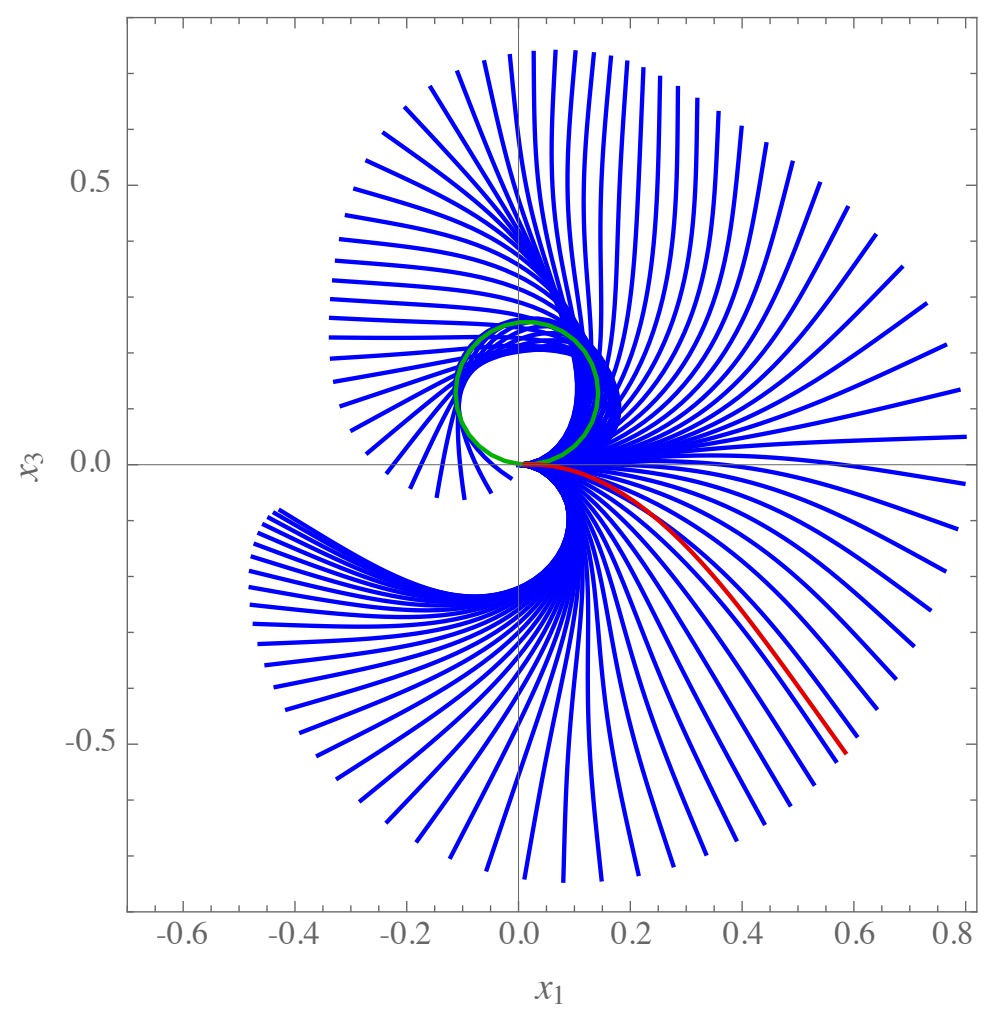

Figure 19. Stroboscopic motion where the initial (green) and final (red) configurations are highlighted.

problems Cazzani et al. (2014, 2015); Greco and Cuomo (2013, 2014b, 2016); Greco et al. (2017) and for dynamics ones Weeger et al. (2013); Yaghoubi et al. (2018)).

Regarding generalizations and future developments, the proposed formulation can easily be adapted for Timoshenko beams (Cazzani et al. 2016; Balobanov and Niiranen 2018) and Cosserat rods (Altenbach et al. 2012, 2013).

The nonlinear nature of the analyzed mechanical system implies that many features that are difficult to be tackled (see, e.g., in the context of nonlinear vibrations Battisti et al. (2017); Chróścielewski et al. (2019); Deü et al. (2008); Lazarus et al. (2012); Thomas et al. (2016), nonlinear motions Boyer et al. (2002); Boyer and Primault (2004) as well as in dynamic stability of mechanical systems Luongo and D'Annibale (2013, 2017); Luongo et al. (2016, 2015); Spagnuolo and Andreaus (2019)). Therefore, having a formulation which is as simple as possible is always an opportunity to pursue. 


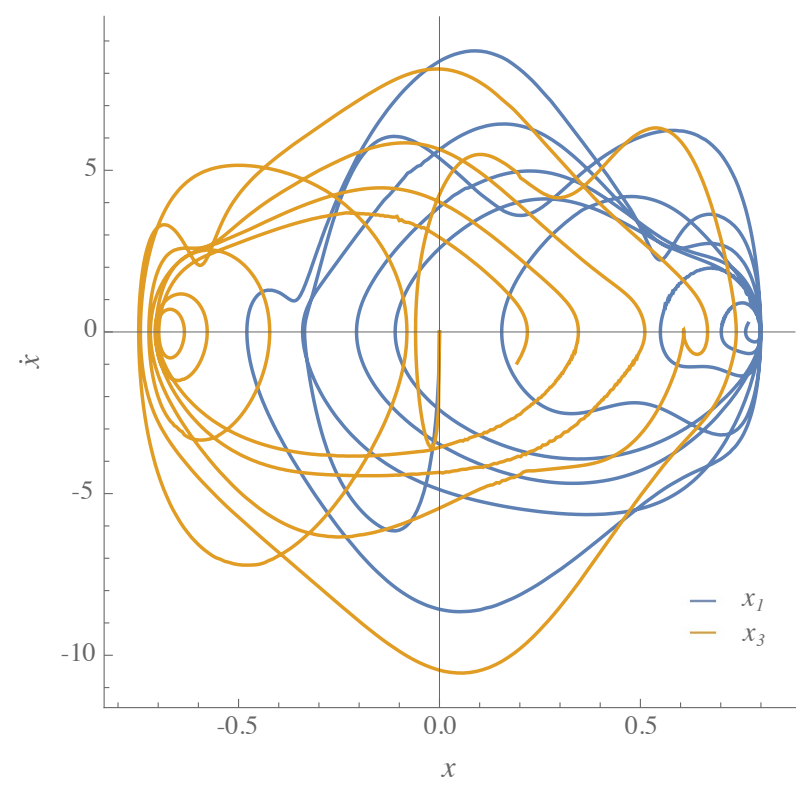

Figure 20. Phase space trajectories of the tip coordinates of the rod.

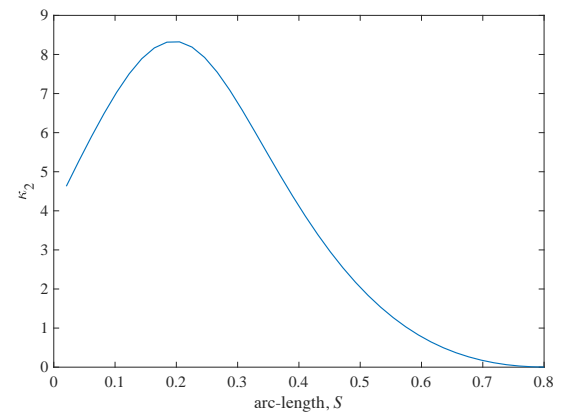

(a) at time $0.12 \mathrm{~s}$

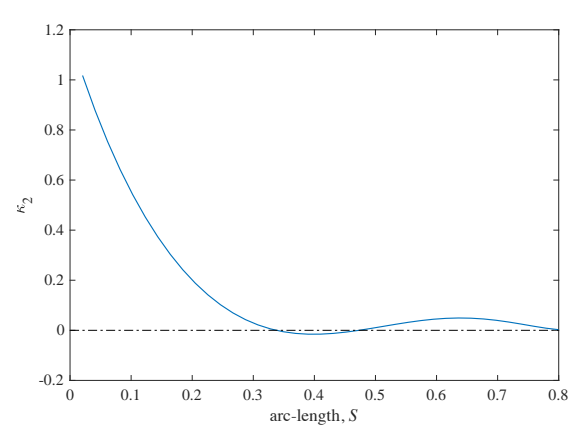

(b) at time $0.38 \mathrm{~s}$

Figure 21. Curvature $\kappa_{2 i}$ along $\boldsymbol{m}_{i}$.

\section{References}

Abali BE, Wu CC and Müller WH (2016) An energy-based method to determine material constants in nonlinear rheology with applications. Continuum Mechanics and Thermodynamics 28(5): 1221-1246. 


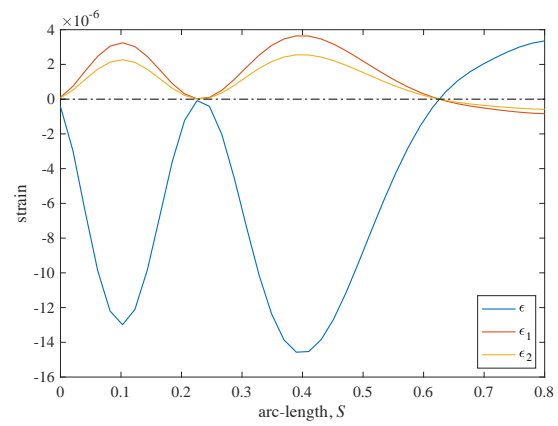

(a) at time $0.12 \mathrm{~s}$

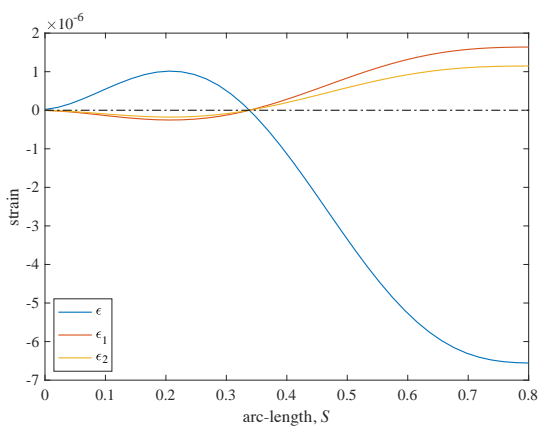

(b) at time $0.38 \mathrm{~s}$

Figure 22. Poisson's effect: strains along $\boldsymbol{e}_{i}, \boldsymbol{m}_{i}$, and $\boldsymbol{n}_{i}$.

Alessandroni S, dell'Isola F and Porfiri M (2002) A revival of electric analogs for vibrating mechanical systems aimed to their efficient control by PZT actuators. International Journal of Solids and Structures 39(20): 5295-5324.

Alibert JJ, Seppecher P and dell'Isola F (2003) Truss modular beams with deformation energy depending on higher displacement gradients. Mathematics and Mechanics of Solids 8(1): 5173.

Altenbach H, Bîrsan M and Eremeyev VA (2012) On a thermodynamic theory of rods with two temperature fields. Acta Mechanica 223(8): 1583-1596.

Altenbach H, Bîrsan M and Eremeyev VA (2013) Cosserat-type rods. In: Generalized Continua from the Theory to Engineering Applications. Springer, pp. 179-248.

Altenbach H and Eremeyev V (2015) On the constitutive equations of viscoelastic micropolar plates and shells of differential type. Mathematics and Mechanics of Complex Systems 3(3): 273-283.

Andreaus U, dell'Isola F and Porfiri M (2004) Piezoelectric passive distributed controllers for beam flexural vibrations. Journal of Vibration and Control 10(5): 625-659.

Avella M, Dell'Erba R and Martuscelli E (1996) Fiber reinforced polypropylene: Influence of iPP molecular weight on morphology, crystallization, and thermal and mechanical properties. Polymer composites 17(2): 288-299.

Balobanov V and Niiranen J (2018) Locking-free variational formulations and isogeometric analysis for the Timoshenko beam models of strain gradient and classical elasticity. Computer Methods in Applied Mechanics and Engineering 339: 137-159.

Barchiesi E, Eugster SR, Placidi L and dell'Isola F (2019a) Pantographic beam: a complete second gradient 1D-continuum in plane. Zeitschrift für angewandte Mathematik und Physik 70(5): 135. 
Barchiesi E and Khakalo S (2019) Variational asymptotic homogenization of beam-like square lattice structures. Mathematics and Mechanics of Solids 24(10): 3295-3318.

Barchiesi E, Spagnuolo M and Placidi L (2019b) Mechanical metamaterials: a state of the art. Mathematics and Mechanics of Solids 24(1): 212-234.

Baroudi D, Giorgio I, Battista A, Turco E and Igumnov LA (2019) Nonlinear dynamics of uniformly loaded Elastica: Experimental and numerical evidence of motion around curled stable equilibrium configurations. ZAMM - Zeitschrift für Angewandte Mathematik und Mechanik 99(7): 20 pages. DOI:10.1002/zamm.201800121. URL http: / / dx . doi .org/ 10.1002 / zamm. 201800121.

Battisti U, Berchio E, Ferrero A and Gazzola F (2017) Energy transfer between modes in a nonlinear beam equation. Journal de Mathématiques Pures et Appliquées 108(6): 885-917.

Berezovski A, Yildizdag ME and Scerrato D (2018) On the wave dispersion in microstructured solids. Continuum Mechanics and Thermodynamics : 1-20.

Bergou M, Wardetzky M, Robinson S, Audoly B and Grinspun E (2008) Discrete elastic rods. In: ACM transactions on graphics (TOG), volume 27. ACM, p. 63.

Bersani A, dell' Isola F and Seppecher P (2019) Lagrange multipliers in infinite dimensional spaces, examples of application. In: Altenbach H., Öchsner A. (eds) Encyclopedia of Continuum Mechanics. Springer, Berlin, Heidelberg.

Boyer F, Glandais N and Khalil W (2002) Flexible multibody dynamics based on a non-linear Euler-Bernoulli kinematics. International journal for numerical methods in engineering 54(1): 27-59.

Boyer F and Primault D (2004) Finite element of slender beams in finite transformations: a geometrically exact approach. International Journal for Numerical Methods in Engineering 59(5): 669-702.

Brezov DS, Mladenova CD and Mladenov IM (2013) New perspective on the gimbal lock problem. In: AIP Conference Proceedings, volume 1570. AIP, pp. 367-374.

Cazzani A, Malagù M and Turco E (2014) Isogeometric analysis of plane-curved beams. Mathematics and Mechanics of Solids 21(5): 562-577. DOI:10.1177/1081286514531265. URL http://dx.doi.org/10.1177/1081286514531265.

Cazzani A, Malagù M, Turco E and Stochino F (2015) Constitutive models for strongly curved beams in the frame of isogeometric analysis. Mathematics and Mechanics of Solids 21(2): 182-209. DOI:10.1177/1081286515577043. URL http://dx.doi.org/10.1177/ 1081286515577043.

Cazzani A, Stochino F and Turco E (2016) An analytical assessment of finite element and isogeometric analyses of the whole spectrum of Timoshenko beams. ZAMM-Zeitschrift für Angewandte Mathematik und Mechanik 96(10): 1220-1244.

Chróścielewski J, Schmidt R and Eremeyev VA (2019) Nonlinear finite element modeling of vibration control of plane rod-type structural members with integrated piezoelectric patches. Continuum Mechanics and Thermodynamics 31(1): 147-188.

Coleman BD, Dill EH, Lembo M, Lu Z and Tobias I (1993) On the dynamics of rods in the theory of Kirchhoff and Clebsch. Archive for rational mechanics and analysis 121(4): 339-359. 
Della Corte A, dell' Isola F, Esposito R and Pulvirenti M (2017) Equilibria of a clamped Euler beam (Elastica) with distributed load: Large deformations. Mathematical Models and Methods in Applied Sciences 27(08): 1391-1421.

dell'Isola F, Andreaus U and Placidi L (2015) At the origins and in the vanguard of peridynamics, non-local and higher-gradient continuum mechanics: An underestimated and still topical contribution of Gabrio Piola. Mathematics and Mechanics of Solids 20(8): 887-928.

dell'Isola F and Di Cosmo F (2018) Lagrange multipliers in infinite-dimensional systems, methods of. In: Altenbach H., Öchsner A. (eds) Encyclopedia of Continuum Mechanics. Springer, Berlin, Heidelberg.

dell'Isola F, Giorgio I, Pawlikowski M and Rizzi NL (2016) Large deformations of planar extensible beams and pantographic lattices: heuristic homogenization, experimental and numerical examples of equilibrium. Proceedings of the Royal Society A: Mathematical, Physical and Engineering Sciences 472(2185): 20150790.

dell'Isola F, Porfiri M and Vidoli S (2003) Piezo-ElectroMechanical (PEM) structures: passive vibration control using distributed piezoelectric transducers. Comptes Rendus Mecanique 331(1): 69-76.

dell'Isola F, Seppecher P, Alibert JJ et al. (2019) Pantographic metamaterials: an example of mathematically driven design and of its technological challenges. Continuum Mechanics and Thermodynamics 31(4): 851-884.

Deü JF, Galucio AC and Ohayon R (2008) Dynamic responses of flexible-link mechanisms with passive/active damping treatment. Computers \& structures 86(3-5): 258-265.

Eugster SR (2015) Geometric continuum mechanics and induced beam theories, volume 75. Springer.

Eugster SR, Hesch C, Betsch P and Glocker C (2014) Director-based beam finite elements relying on the geometrically exact beam theory formulated in skew coordinates. International Journal for Numerical Methods in Engineering 97(2): 111-129.

Ferrarotti A, Piccardo G and Luongo A (2017) A novel straightforward dynamic approach for the evaluation of extensional modes within GBT 'cross-section analysys'. Thin-Walled Structures 114: 52-69.

Greco L and Cuomo M (2013) B-spline interpolation of Kirchhoff-Love space rods. Computer Methods in Applied Mechanics and Engineering 256: 251 - 269. DOI:https://doi.org/10.1016/ j.cma.2012.11.017. URL http://www.sciencedirect.com/science/article/ pii/s0045782512003660.

Greco L and Cuomo M (2014a) Consistent tangent operator for an exact Kirchhoff rod model. Continuum Mechanics and Thermodynamics 27(4-5): 861-877. DOI:10.1007/ s00161-014-0361-x. URL http://dx.doi.org/10.1007/s00161-014-0361-x.

Greco L and Cuomo M (2014b) An implicit G1 multi patch B-spline interpolation for KirchhoffLove space rod. Computer Methods in Applied Mechanics and Engineering 269: 173 - 197. DOI:https://doi.org/10.1016/j.cma.2013.09.018. URL http://www.sciencedirect. $\mathrm{com} / \mathrm{science/article/pii/s0045782513002454.}$ 
Greco L and Cuomo M (2016) An isogeometric implicit G1 mixed finite element for Kirchhoff space rods. Computer Methods in Applied Mechanics and Engineering 298: 325 - 349. DOI: https://doi.org/10.1016/j.cma.2015.06.014. URL http://www. sciencedirect.com/ science/article/pii/s0045782515002066.

Greco L, Cuomo M, Contrafatto L and Gazzo S (2017) An efficient blended mixed B-spline formulation for removing membrane locking in plane curved Kirchhoff rods. Computer Methods in Applied Mechanics and Engineering 324: 476 - 511. DOI:https://doi.org/10.1016/ j.cma.2017.06.032. URL http://www. sciencedirect.com/science/article/ pii/so045782517305467.

Jawed MK, Novelia A and O'Reilly OM (2018) A primer on the kinematics of discrete elastic rods. Springer.

Karathanasopoulos N, Reda H and Ganghoffer JF (2017) Designing two-dimensional metamaterials of controlled static and dynamic properties. Computational Materials Science 138: 323-332.

Laudato M and Barchiesi E (2019) Non-linear dynamics of pantographic fabrics: Modelling and numerical study. In: Wave Dynamics, Mechanics and Physics of Microstructured Metamaterials. Springer, pp. 241-254.

Lazarus A, Thomas O and Deü JF (2012) Finite element reduced order models for nonlinear vibrations of piezoelectric layered beams with applications to NEMS. Finite Elements in Analysis and Design 49(1): 35-51.

Lekszycki T, Olhoff N and Pedersen JJ (1992) Modelling and identification of viscoelastic properties of vibrating sandwich beams. Composite structures 22(1): 15-31.

Lossouarn B, Deü JF and Aucejo M (2015) Multimodal vibration damping of a beam with a periodic array of piezoelectric patches connected to a passive electrical network. Smart Materials and Structures 24(11): 115037.

Lossouarn B, Deü JF, Aucejo M and Cunefare KA (2016) Multimodal vibration damping of a plate by piezoelectric coupling to its analogous electrical network. Smart Materials and Structures 25(11): 115042.

Luongo A and D'Annibale F (2013) Double zero bifurcation of non-linear viscoelastic beams under conservative and non-conservative loads. International Journal of Non-Linear Mechanics 55: $128-139$.

Luongo A and D'Annibale F (2017) Nonlinear hysteretic damping effects on the post-critical behaviour of the visco-elastic Beck's beam. Mathematics and Mechanics of Solids 22(6): 1347-1365.

Luongo A, Ferretti M and D'Annibale F (2016) Paradoxes in dynamic stability of mechanical systems: investigating the causes and detecting the nonlinear behaviors. SpringerPlus 5(1): 60.

Luongo A, Ferretti M and Seyranian A (2015) Effects of damping on the stability of the compressed Nicolai beam. Mathematics and Mechanics of Complex Systems 3(1): 1-26.

Luongo A and Zulli D (2013) Mathematical models of beams and cables. John Wiley \& Sons.

Nejadsadeghi N, De Angelo M, Drobnicki R, Lekszycki T, dell'Isola F and Misra A (2019a) Parametric experimentation on pantographic unit cells reveals local extremum configuration. 
Experimental Mechanics : 1-13.

Nejadsadeghi N, Placidi L, Romeo M and Misra A (2019b) Frequency band gaps in dielectric granular metamaterials modulated by electric field. Mechanics Research Communications 95: 96-103.

Niiranen J, Balobanov V, Kiendl J and Hosseini SB (2019) Variational formulations, model comparisons and numerical methods for Euler-Bernoulli micro-and nano-beam models. Mathematics and Mechanics of Solids 24(1): 312-335.

Pai PF (2011) Geometrically exact beam theory without Euler angles. International Journal of Solids and Structures 48(21): 3075-3090.

Piccardo G, Ranzi G and Luongo A (2014) A direct approach for the evaluation of the conventional modes within the GBT formulation. Thin-Walled Structures 74: 133-145.

Pideri C and Seppecher P (2006) Asymptotics of a non-planar rod in non-linear elasticity. Asymptotic Analysis 48(1, 2): 33-54.

Placidi L, dell' Isola F and Barchiesi E (2020) Heuristic homogenization of Euler and pantographic beams. In: Mechanics of Fibrous Materials and Applications. Springer, pp. 123-155.

Reda H, Karathanasopoulos N, Elnady K, Ganghoffer JF and Lakiss H (2018) Mechanics of metamaterials: an overview of recent developments. In: Advances in Mechanics of Microstructured Media and Structures. Springer, pp. 273-296.

Simo JC (1985) A finite strain beam formulation. The three-dimensional dynamic problem. Part I. Computer methods in applied mechanics and engineering 49(1): 55-70.

Simo JC and Vu-Quoc L (1986) A three-dimensional finite-strain rod model. Part II: Computational aspects. Computer methods in applied mechanics and engineering 58(1): 79-116.

Simo JC and Vu-Quoc L (1988) On the dynamics in space of rods undergoing large motionsa geometrically exact approach. Computer methods in applied mechanics and engineering 66(2): 125-161.

Solyaev Y, Lurie S, Barchiesi E and Placidi L (2019) On the dependence of standard and gradient elastic material constants on a field of defects. Mathematics and Mechanics of Solids : doi.org/10.1177/1081286519861827.

Spagnuolo M and Andreaus U (2019) A targeted review on large deformations of planar elastic beams: extensibility, distributed loads, buckling and post-buckling. Mathematics and Mechanics of Solids 24(1): 258-280.

Spagnuolo M, Franciosi P and dell'Isola F (2019) A Green operator-based elastic modeling for two-phase pantographic-inspired bi-continuous materials. International Journal of Solids and Structures DOI:https://doi.org/10.1016/j.ijsolstr.2019.10.018.

Steigmann DJ and Faulkner MG (1993) Variational theory for spatial rods. Journal of Elasticity 33(1): 1-26. DOI:10.1007/bf00042633. URL http://dx.doi.org/10. $1007 / \mathrm{BF} 00042633$.

Thomas O, Sénéchal A and Deü JF (2016) Hardening/softening behavior and reduced order modeling of nonlinear vibrations of rotating cantilever beams. Nonlinear Dynamics 86(2): $1293-1318$. 
Turco E (2018) Discrete is it enough? the revival of Piola-Hencky keynotes to analyze three-dimensional Elastica. Continuum Mechanics and Thermodynamics 30(5): 10391057. DOI:10.1007/s00161-018-0656-4. URL http://dx.doi.org/10.1007/ s00161-018-0656-4.

Turco E, dell'Isola F, Cazzani A and Rizzi NL (2016) Hencky-type discrete model for pantographic structures: numerical comparison with second gradient continuum models. Zeitschrift für angewandte Mathematik und Physik 67(4): 85.

Vangelatos Z, Komvopoulos K and Grigoropoulos CP (2019) Vacancies for controlling the behavior of microstructured three-dimensional mechanical metamaterials. Mathematics and Mechanics of Solids 24(2): 511-524.

Wang CM, Zhang H, Gao RP, Duan WH and Challamel N (2015) Hencky bar-chain model for buckling and vibration of beams with elastic end restraints. International Journal of Structural Stability and Dynamics 15(07): 1540007.

Weeger O, Wever U and Simeon B (2013) Isogeometric analysis of nonlinear Euler-Bernoulli beam vibrations. Nonlinear Dynamics 72(4): 813-835.

Yaghoubi ST, Balobanov V, Mousavi SM and Niiranen J (2018) Variational formulations and isogeometric analysis for the dynamics of anisotropic gradient-elastic euler-bernoulli and shear-deformable beams. European Journal of Mechanics-A/Solids 69: 113-123.

Zhang H, Wang CM and Challamel N (2016a) Buckling and vibration of Hencky bar-chain with internal elastic springs. International Journal of Mechanical Sciences 119: 383-395.

Zhang H, Wang CM, Ruocco E and Challamel N (2016b) Hencky bar-chain model for buckling and vibration analyses of non-uniform beams on variable elastic foundation. Engineering Structures 126: 252-263. 